INDOT Agency Factoids

(System/Comm.)

- Number of signalized intersections- 2570

$-\underline{200}$ connected by fiber

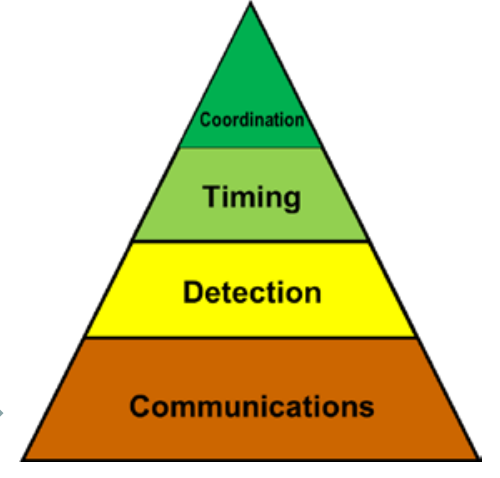

- $\underline{300}$ connected by radio

- $\underline{0}$ connected by twisted pair

- $\underline{225}$ connected by cellular

- 1500 not connected to communication

- Number of engineers/technicians devoted to signals. 8 engineers, 32 Technicians

- Currently 2 vacant engineer positions ( 1 Engineer level, 1 Managing Engineer level)

- Central System Vendor None, Closed loop systems function as central system

- 300 signals collecting high resolution data

- Greater than 10 years collecting high resolution data 


\section{Agency Factoids}

(Detection)

- Length of stop bar detectors on minor movement. $\underline{51 \mathrm{ft}}$

- Use of dilemma zone or other detection on arterial main line. Both stop bar detection and dilemma zone detection used ( 5 seconds in advance of stop bar)

- Detection Technologies used. All in pavement, no above pavement

- "Lane by Lane" or "Lane Group Detection" Lane by lane

- Link to detection standard number scheme

- Detection Testing and Maintenance Practices Testing: Performance based product approvals, Maintenance: Reactive, upon failure

- \# of Signals with Emergency Vehicle Preemption. 100 maint. by others

- \# of Signals with RR Preemption. 75 with pre-empt 


\section{Interstate Diversion}

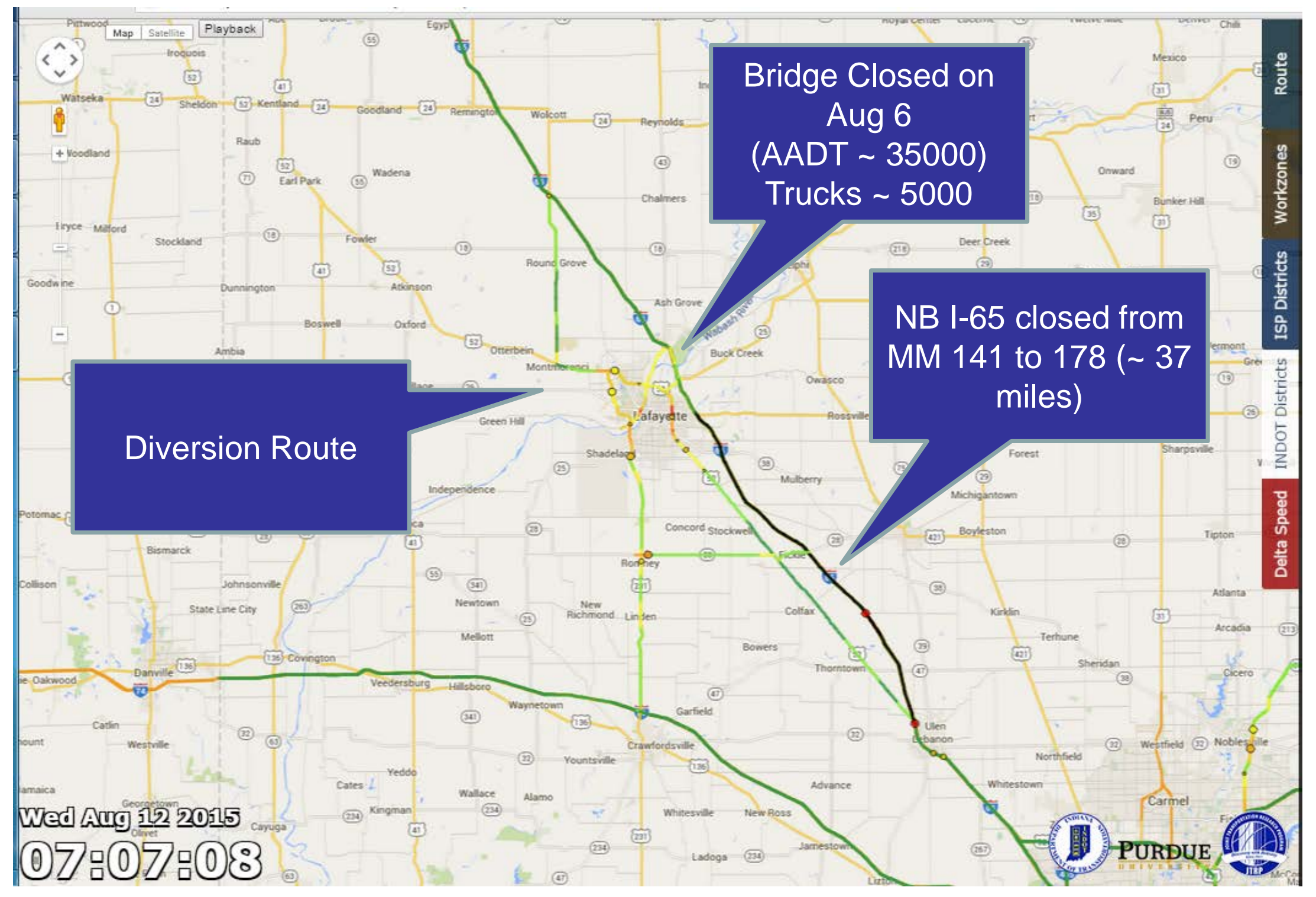


Northbound I-65 Bridge Closure... Repairs In Progress 


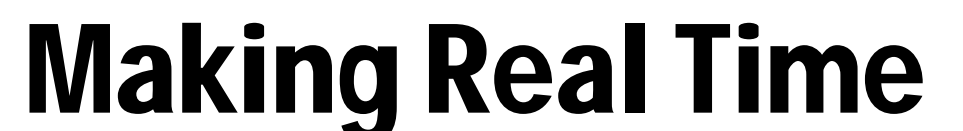

of

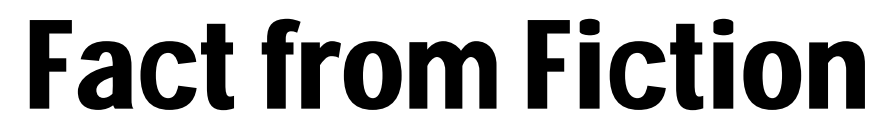

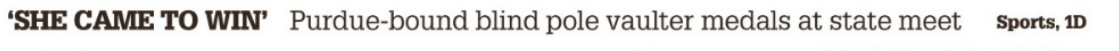

JOURNAL \&OURIER
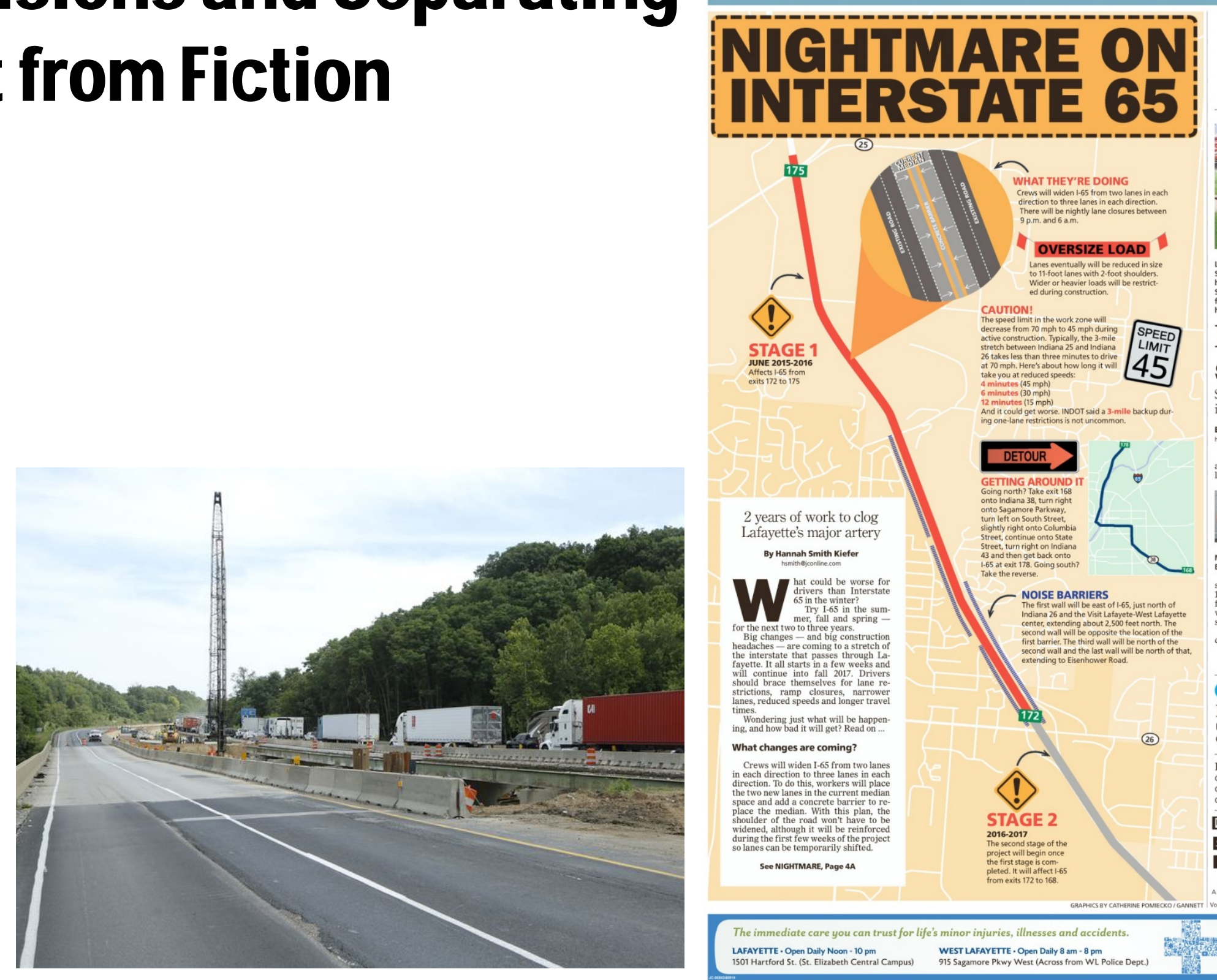

MORE THAN

$\$ 240$

IN COUPONS

INSIDE TODAY!
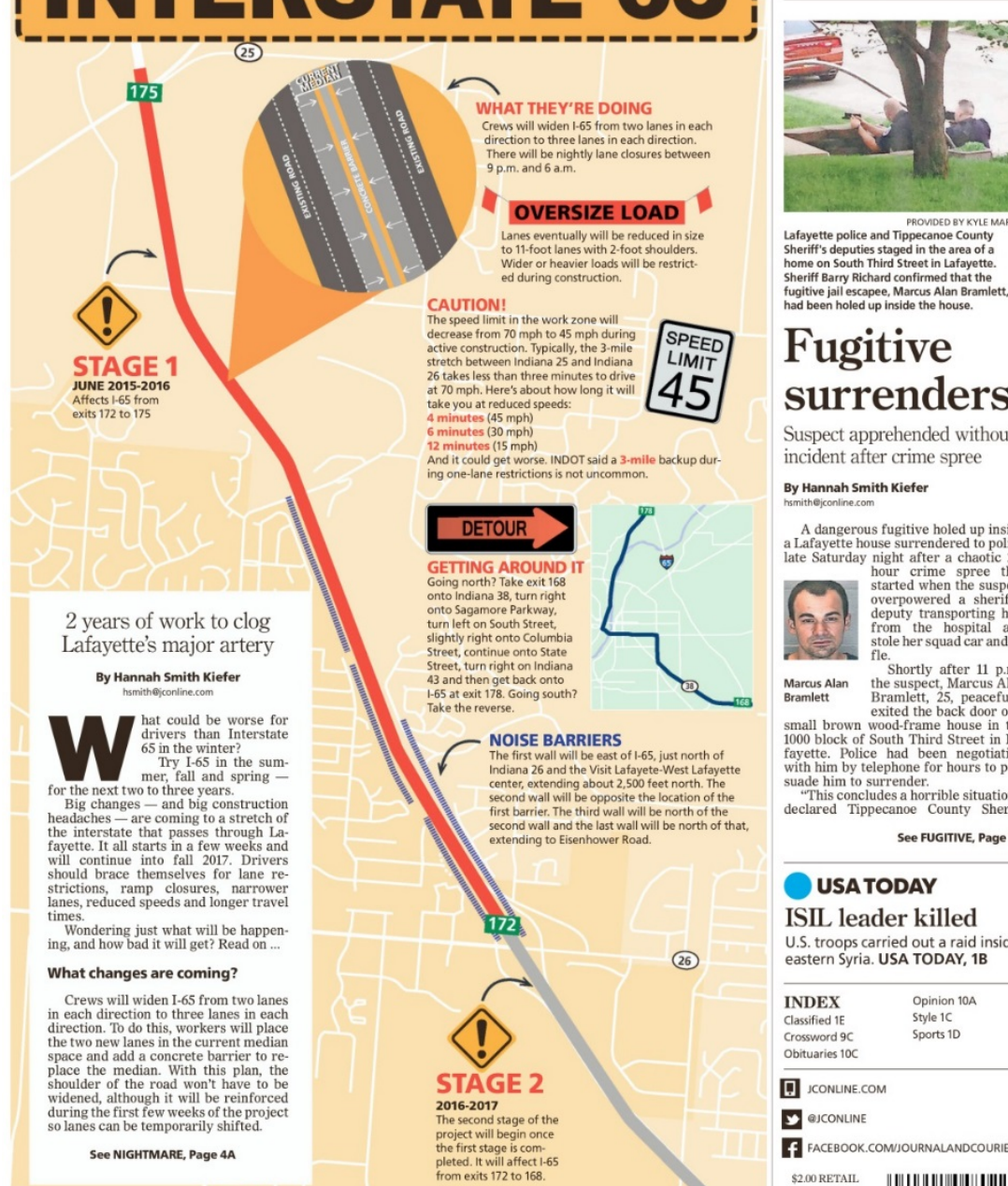

A dangerous fugitive holed up inside
a L Lafayette house surrendered to police

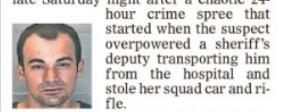

Nace. he.

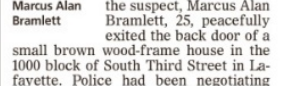

ion block of South Third Street in La
fayeter Police had b ben nogotiating
ivth him by telephone for hours to per-

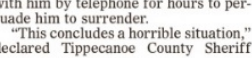

See FUGITIVE, Page 6A

USATOdAY

ISIL leader killed

U.S. troops carried out a raid inside
eastern Syria. USA TODAY, 1B

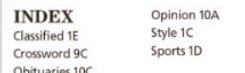

回 JonUne.com

erconur

f $\mathrm{FACE}$

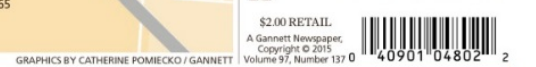




\section{How Bad? Ineffective, Absurd...}

\section{Using Metrics to change the narrative.}

- Reporter drove official

Detour Diary: How bad was the detour really?
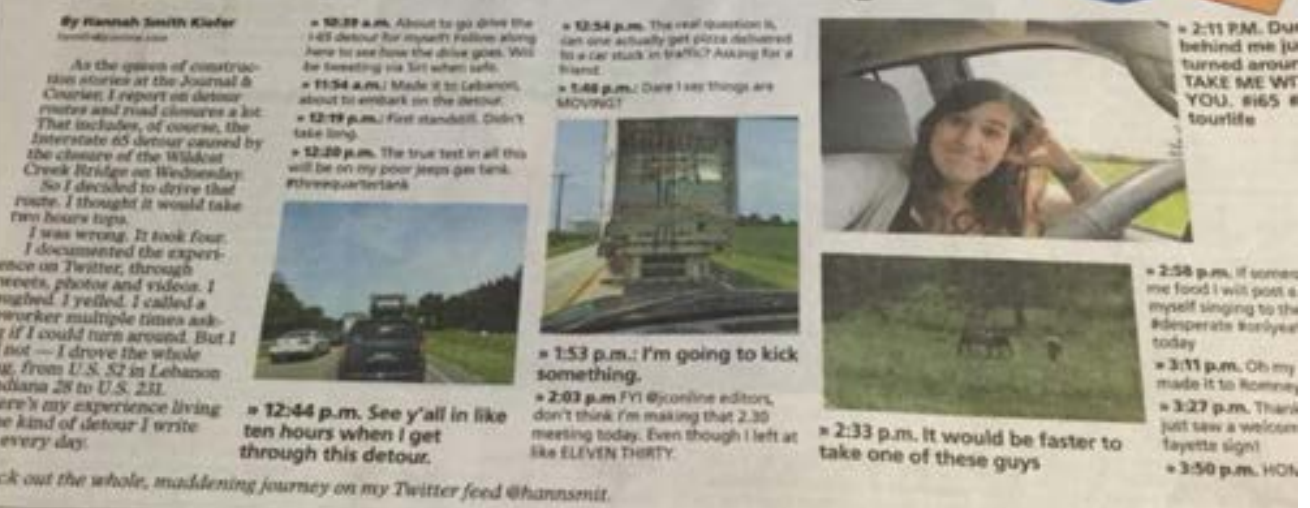

2:33 pms, it would be faster to
take one of these gurs igmil
65 -3sopm detour right after the closure, wrote article for local newspaper

- Took 4 hours to drive $\sim 60$ miles

- Said "Moral of the story is that the INDOT detour route is essentially ineffective."

- "Plan for it to take an absurd amount of time." 


\section{*}

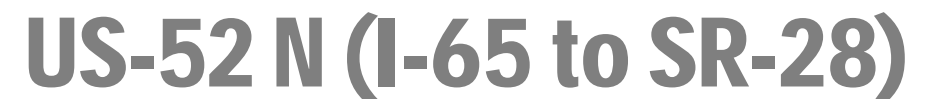

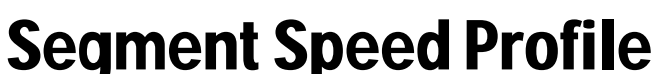

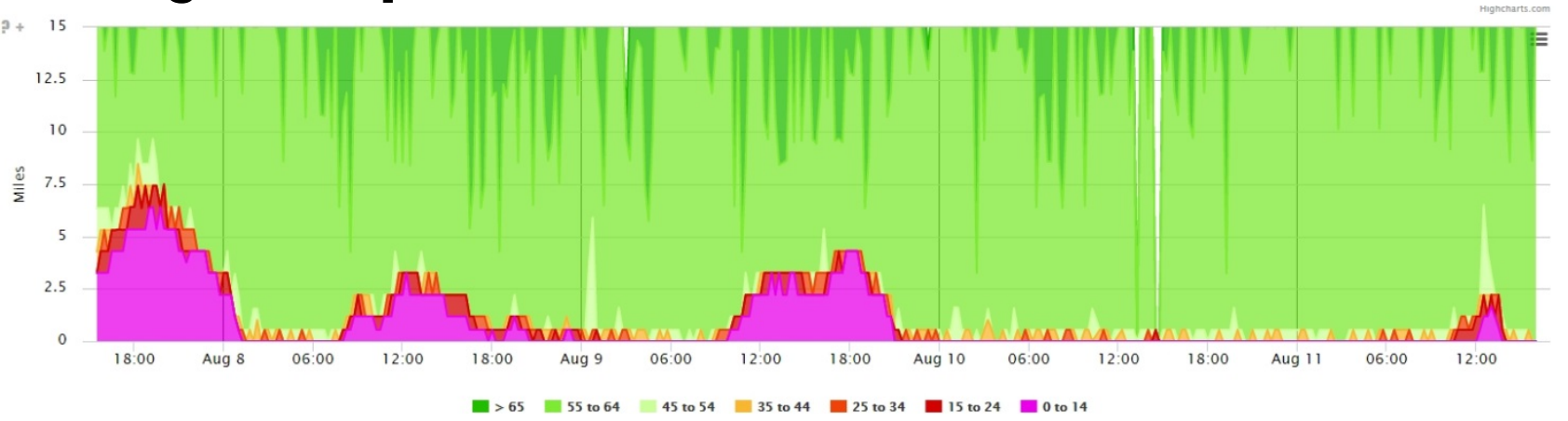

\section{:}

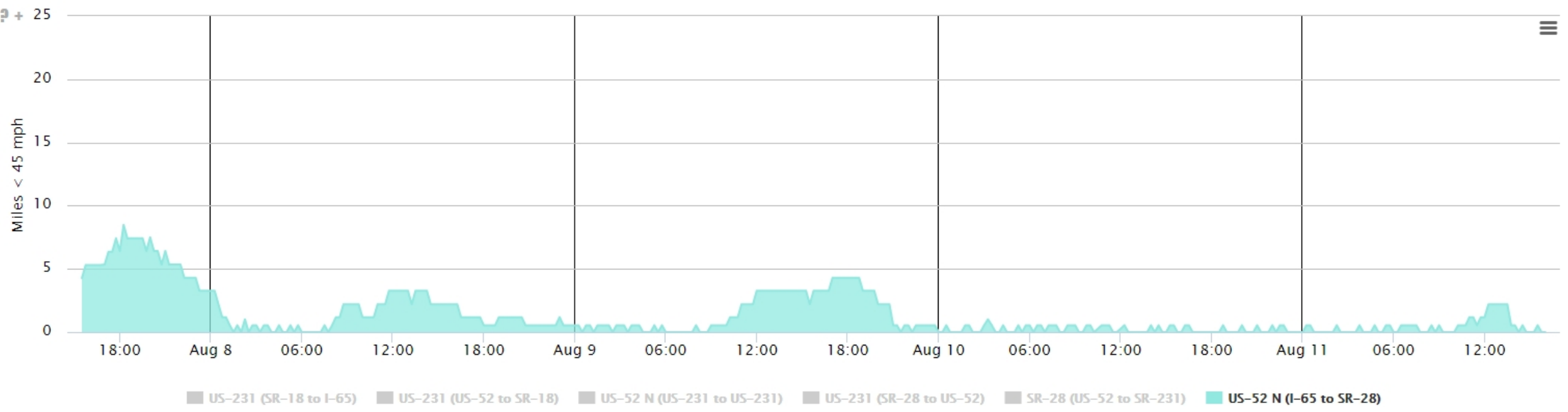




\section{*}

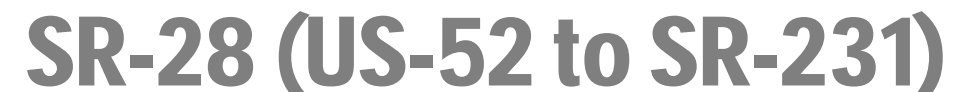

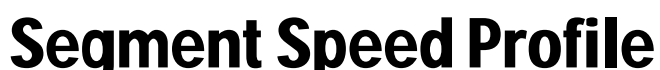

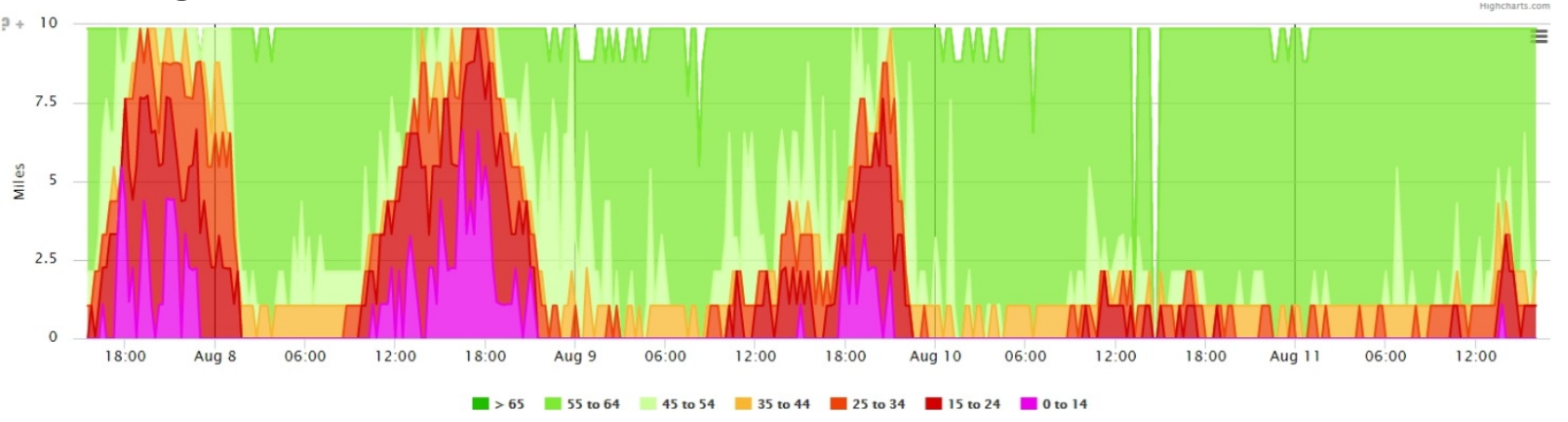

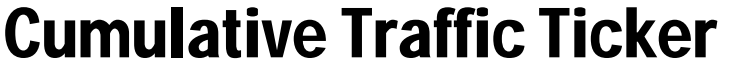

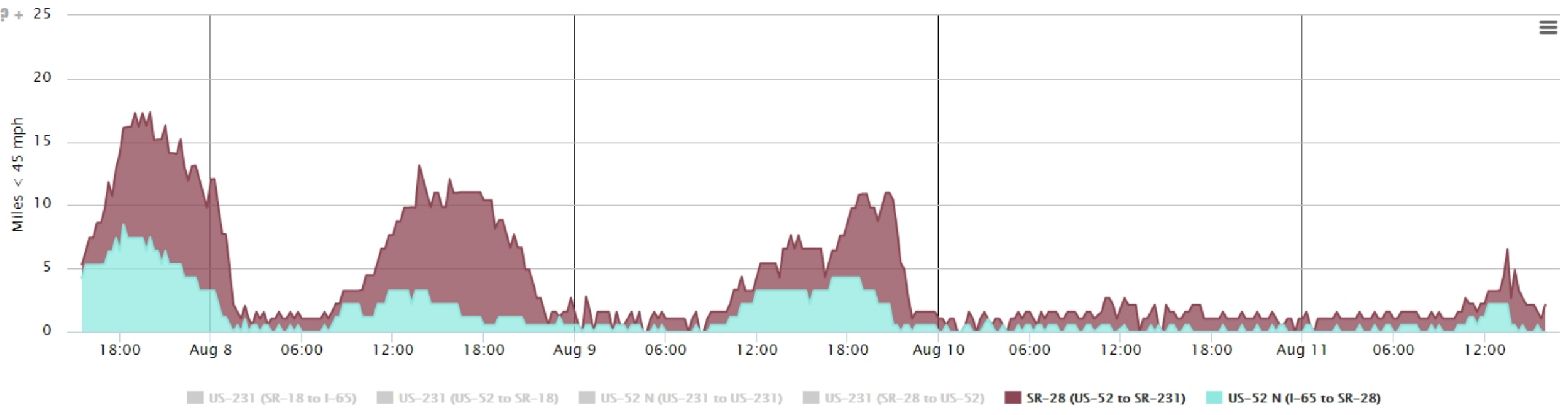




\section{* *}

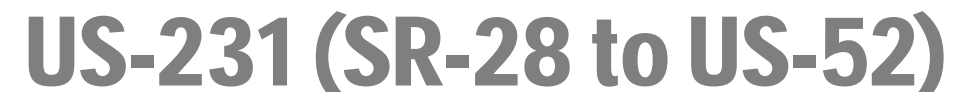

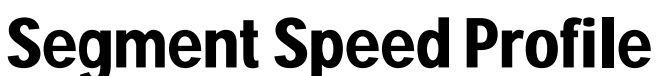

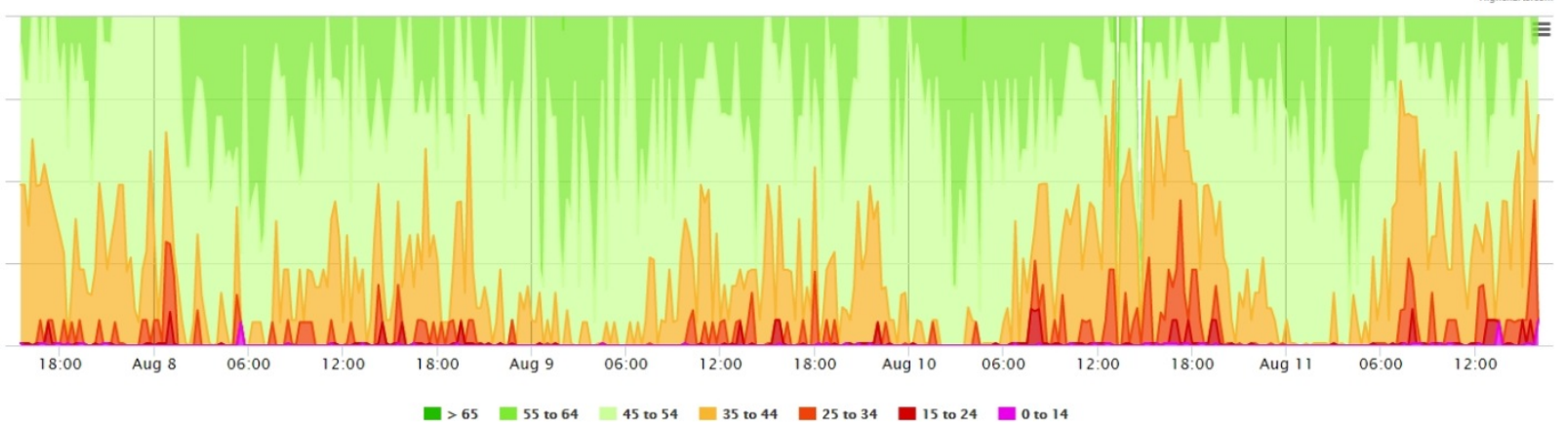

\section{:ค}

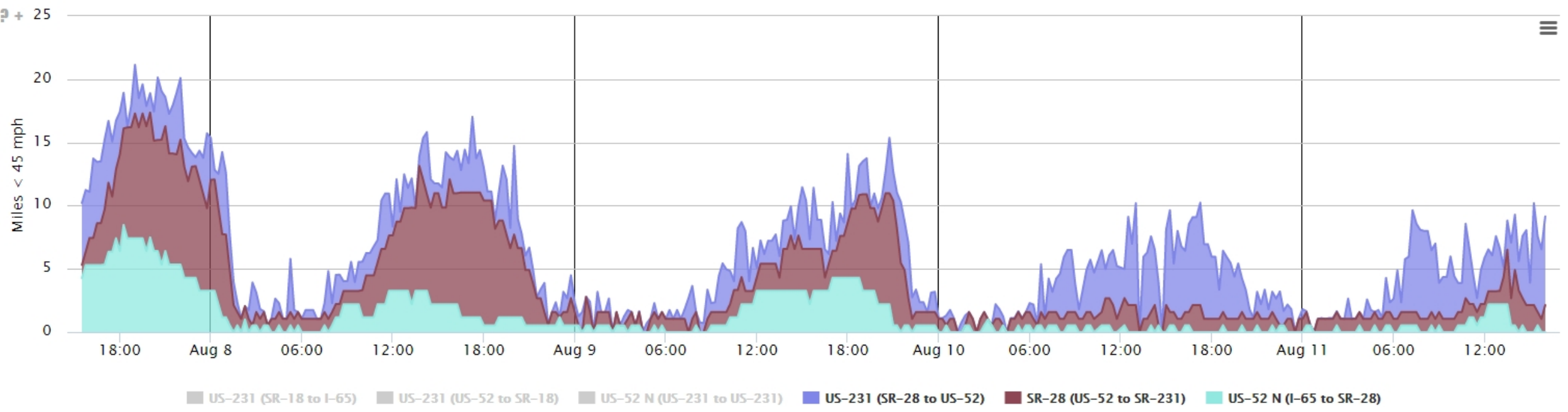




\section{*}

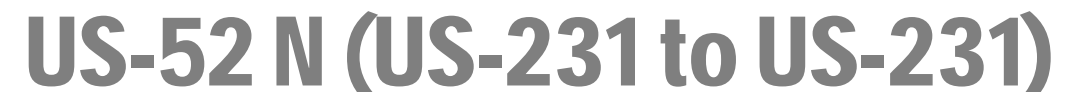

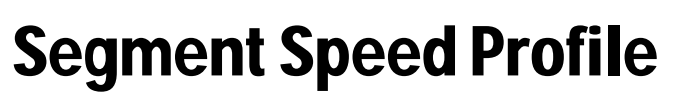

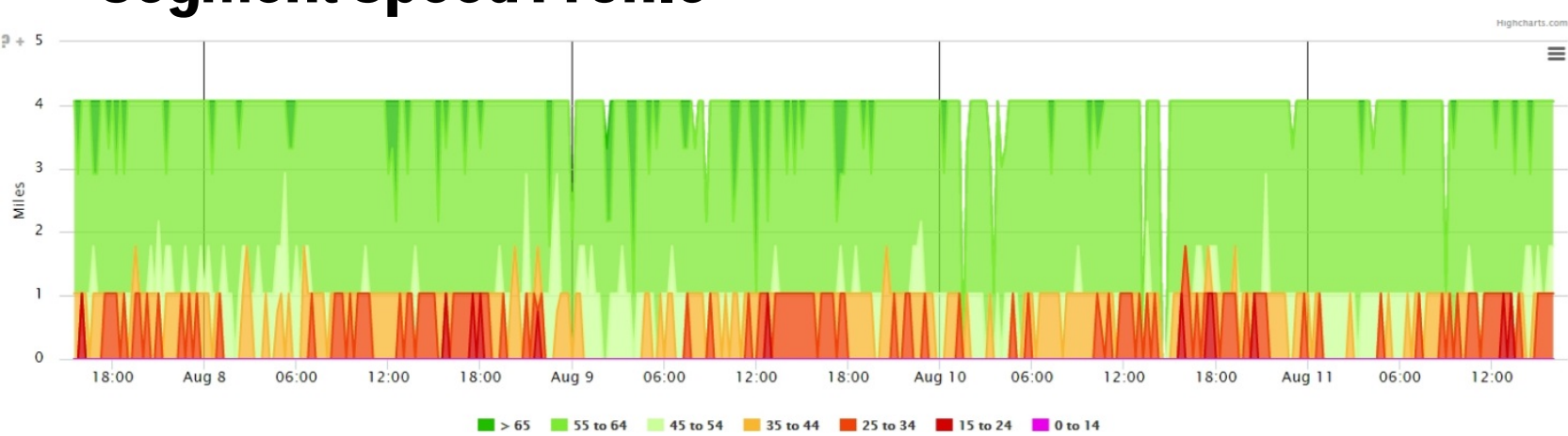

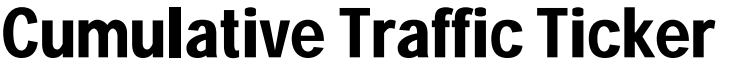

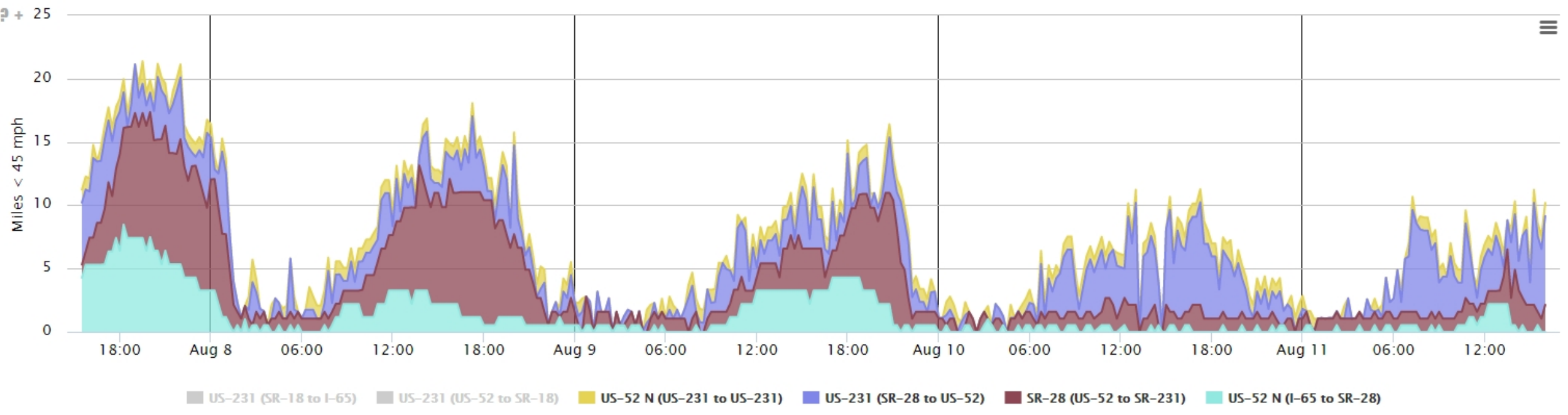




\section{*}

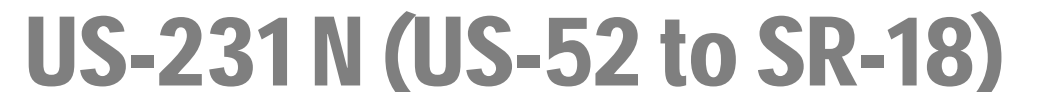

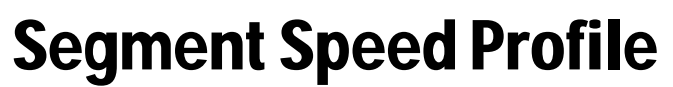

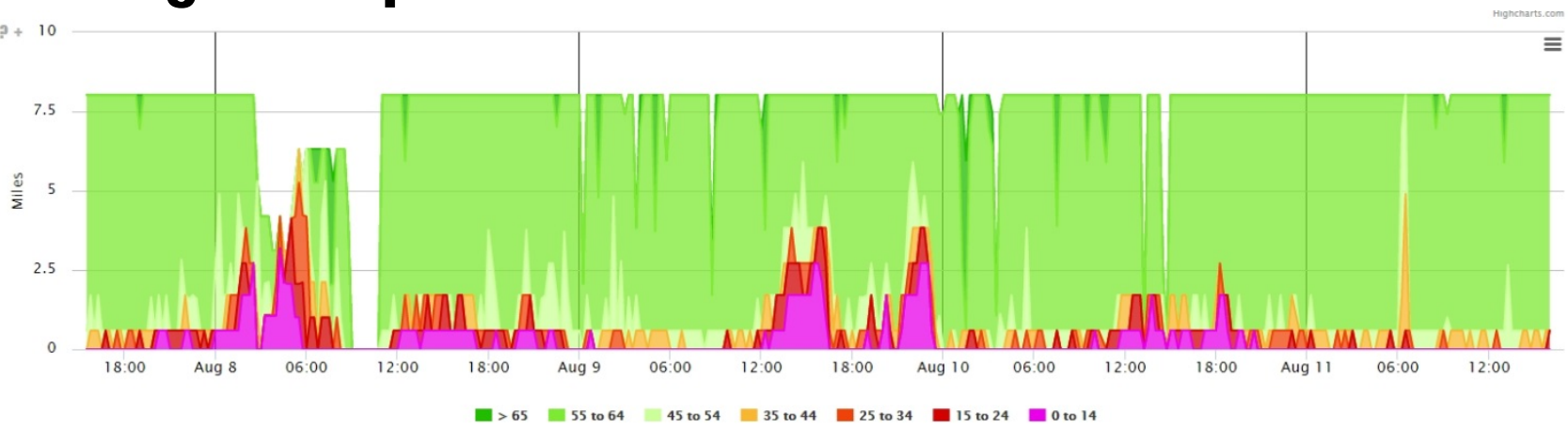

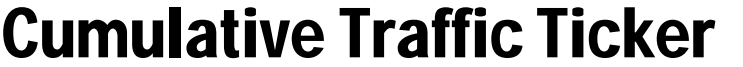

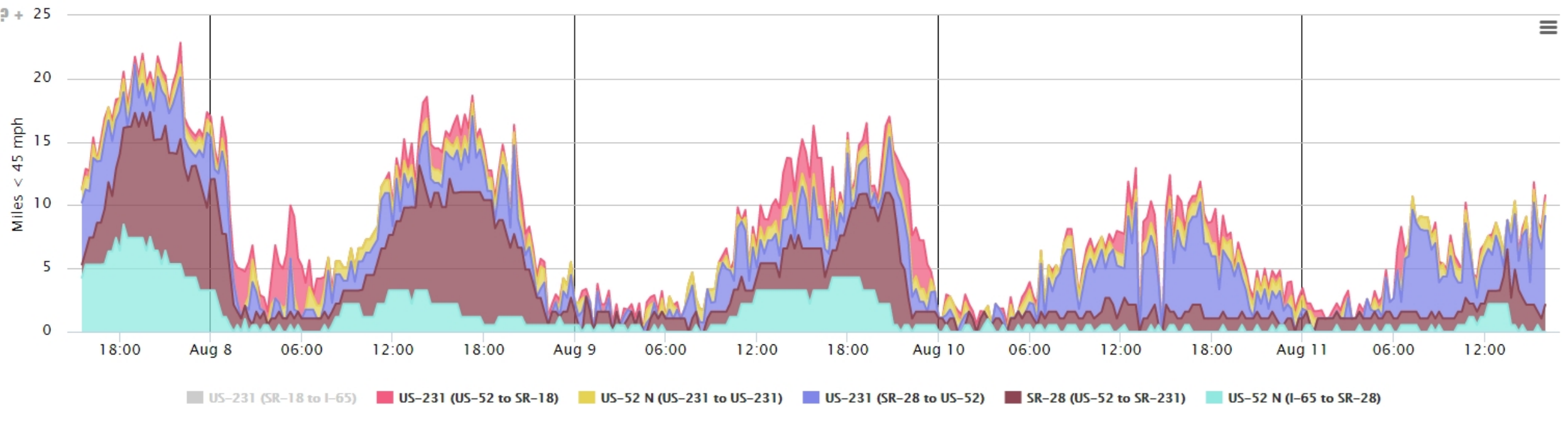




\section{* *}

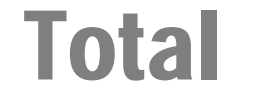

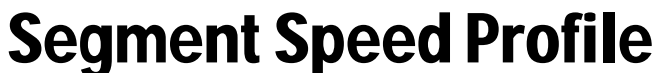

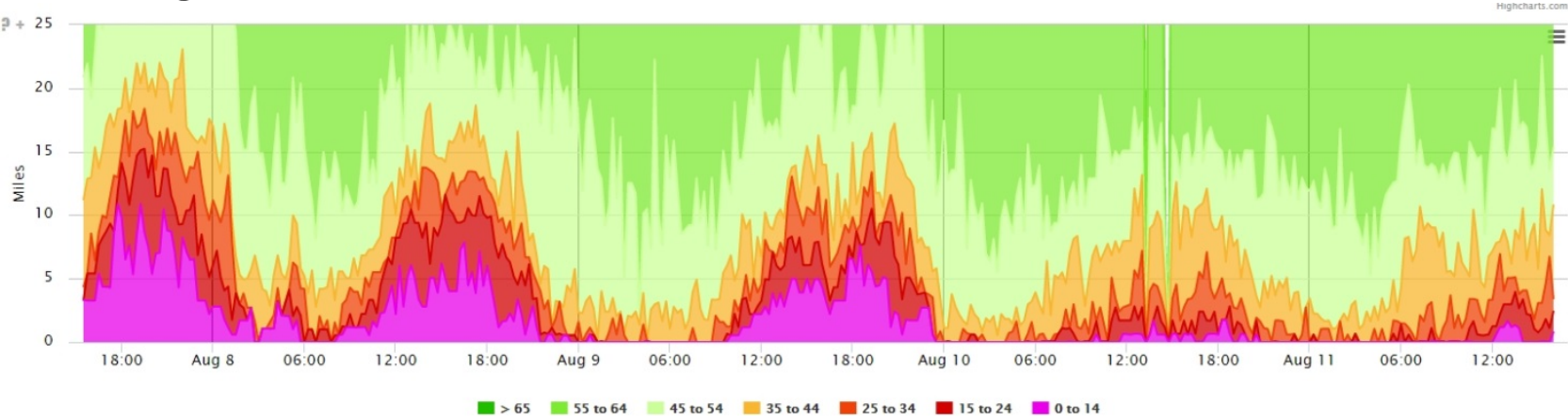

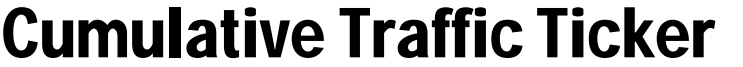

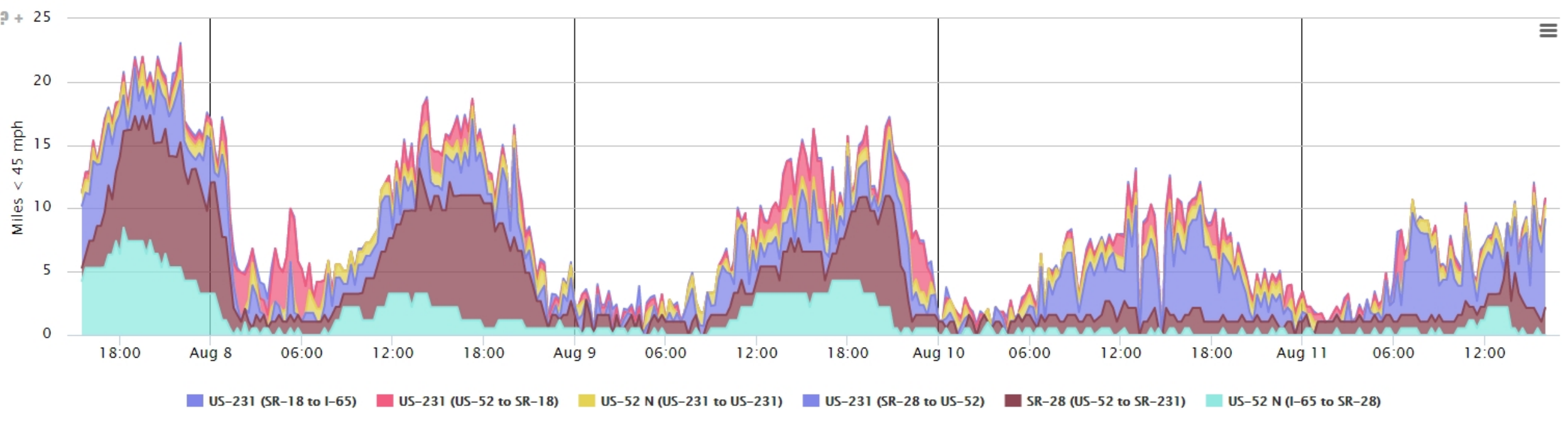




\section{Daily Northbound Volumes -US-231 NB approaching Lafayette}

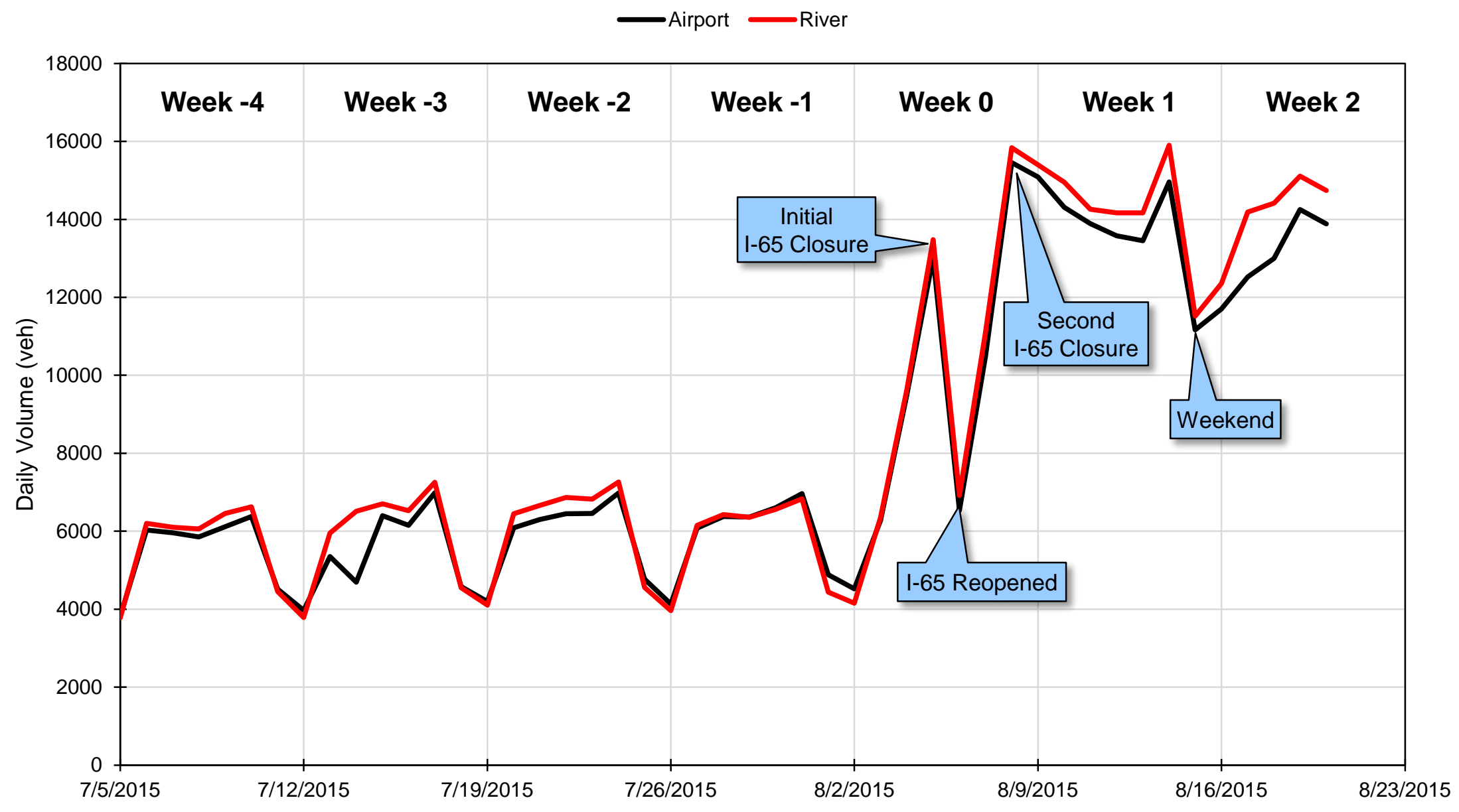




\section{US-231 @ River Road - Typical Week Before and After}

(Week of 7/25 vs. Week of 8/15)

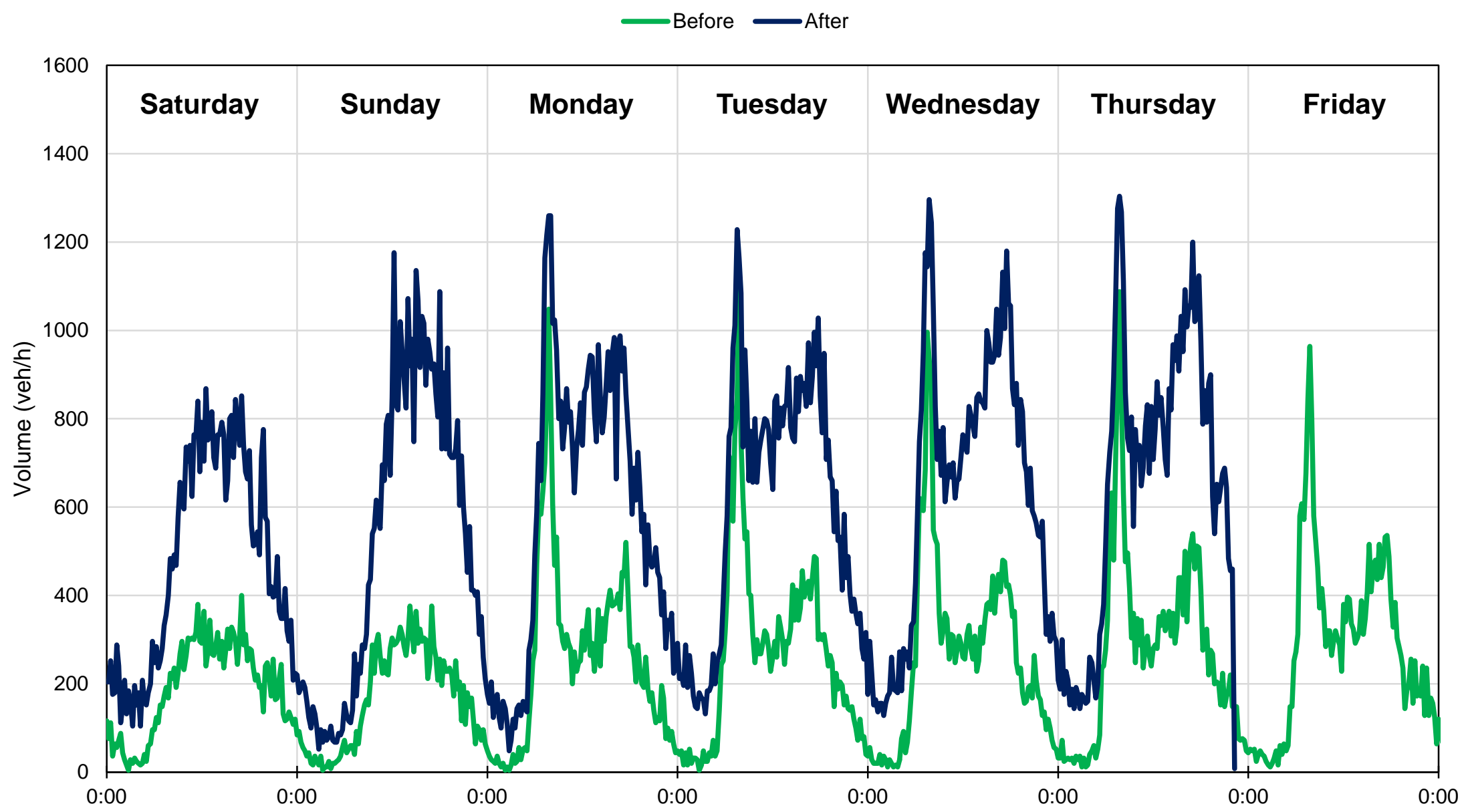




\section{Temp \\ Signals}

\section{Detour Route Dashboard}

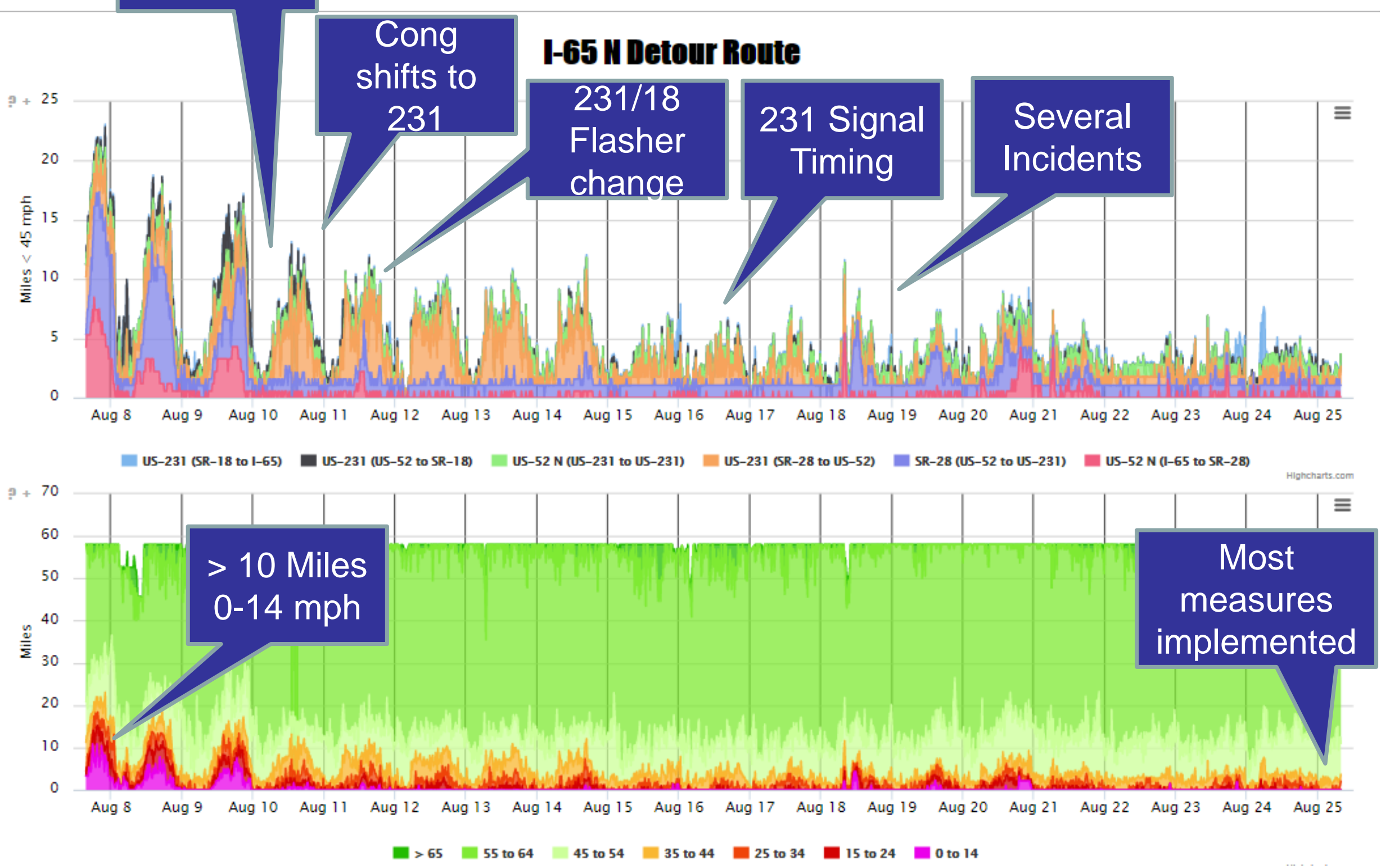




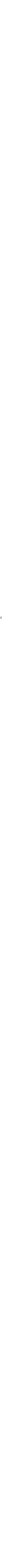




\section{Signalization Impact}
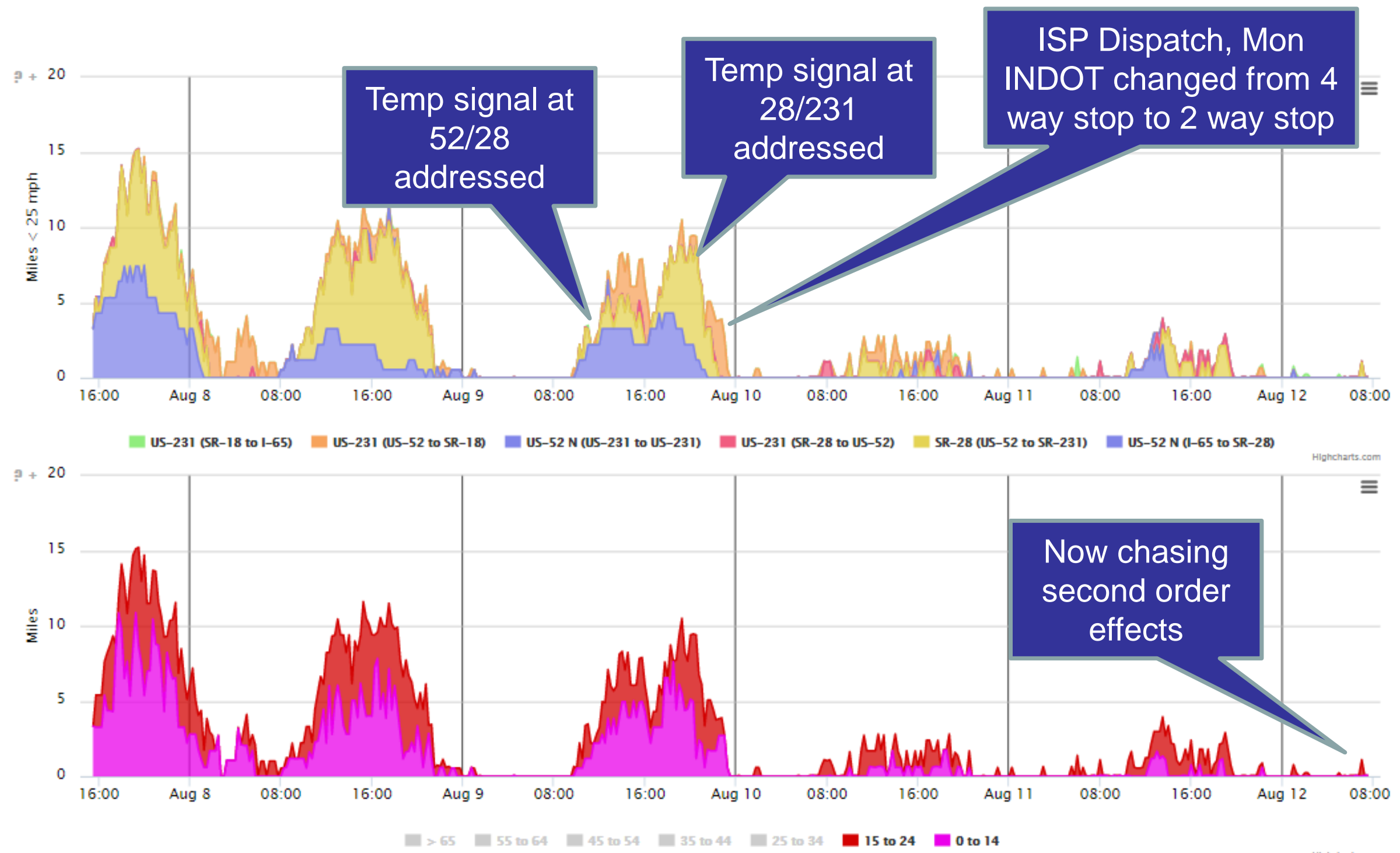


\section{SR 28/US 231 Temporary Signal}

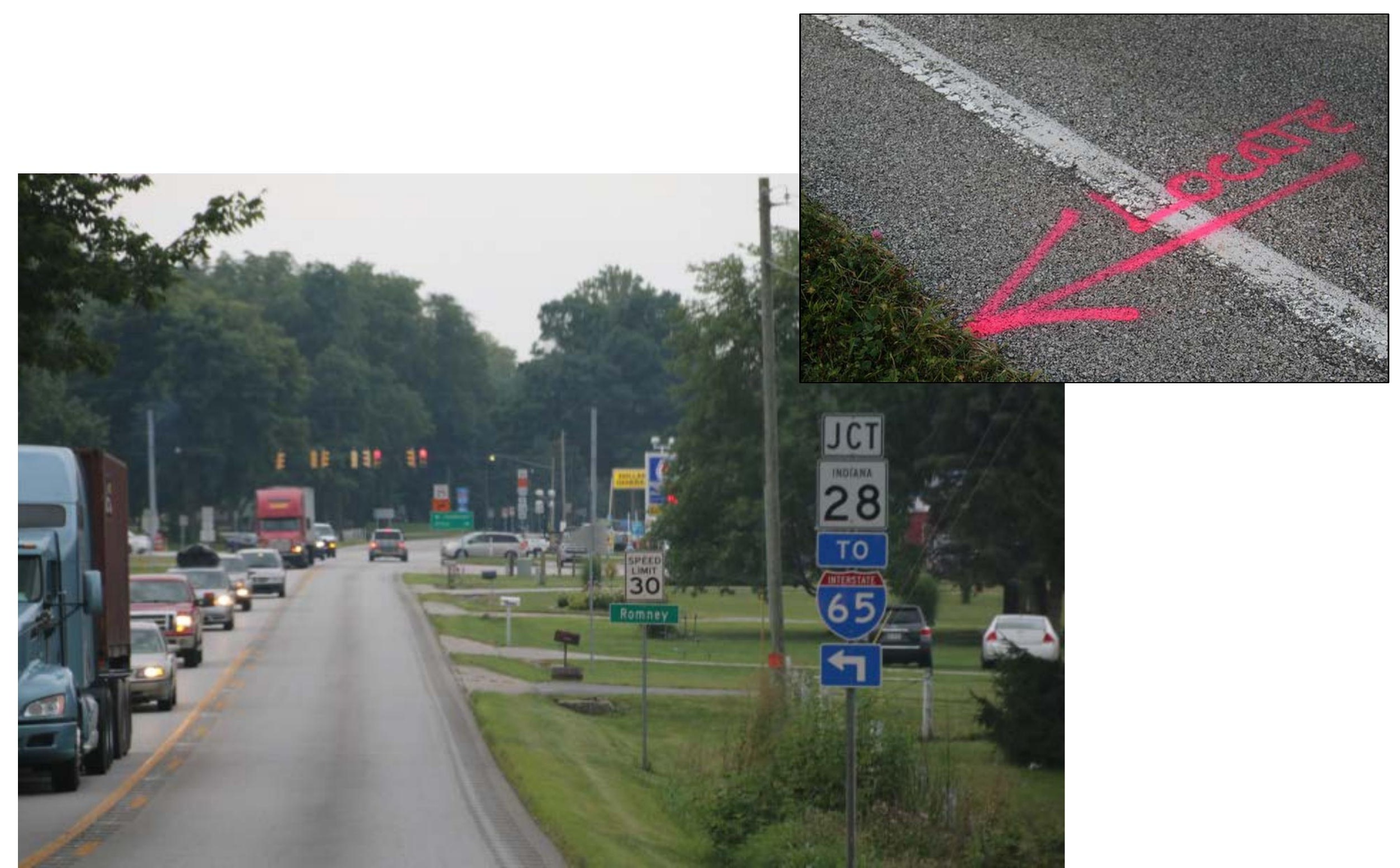


Sunday, August 9, 2015

Ed Cox / Jim Sturdevant @ 231/28

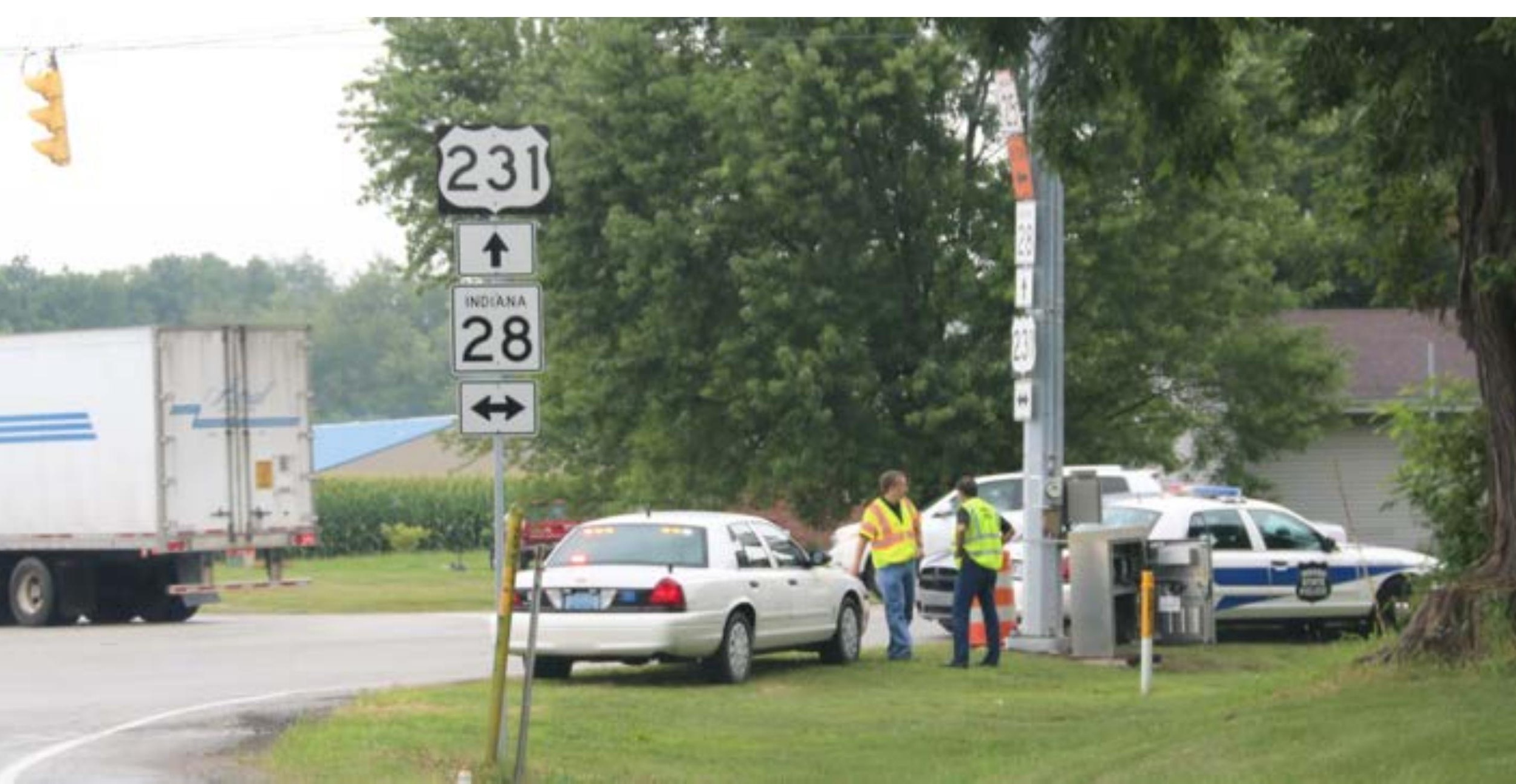




\section{SR 28/US 52 Temporary Signal}

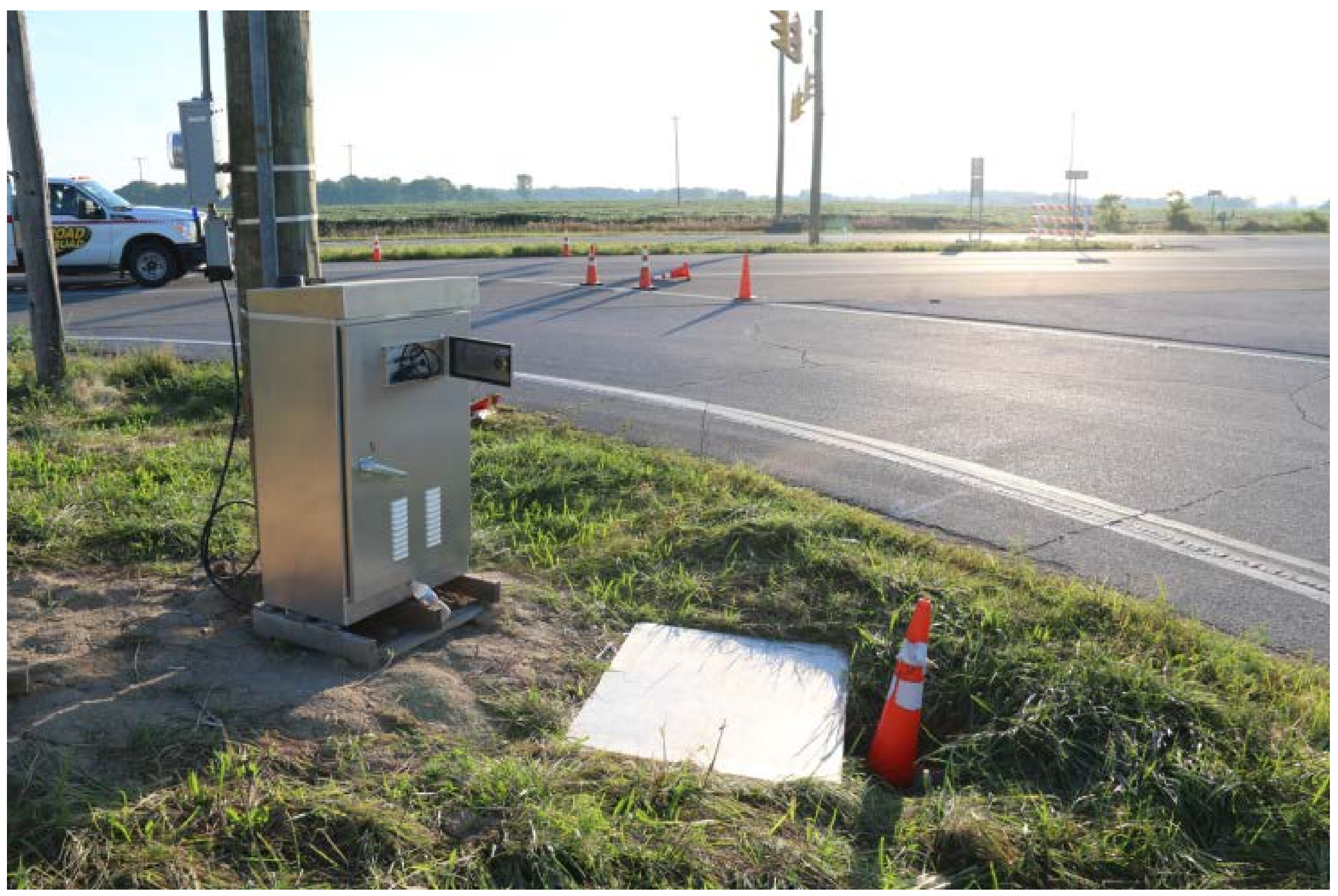




\section{Temporary Signal at US 231 \& SR 28- Romney}

- 2 phase signal

- Installed cell modem for remote access

- Monitored remotely and adjusted splits based on INRIX/Google traffic queuing

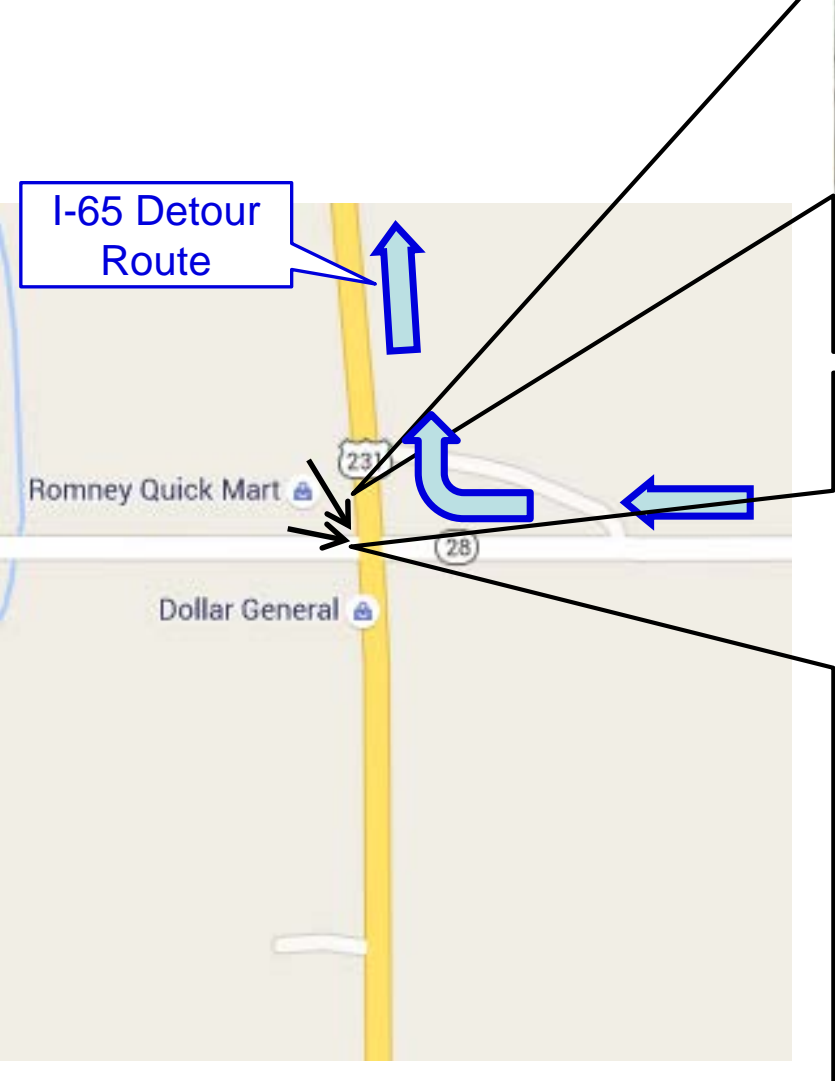

Facing Southeast

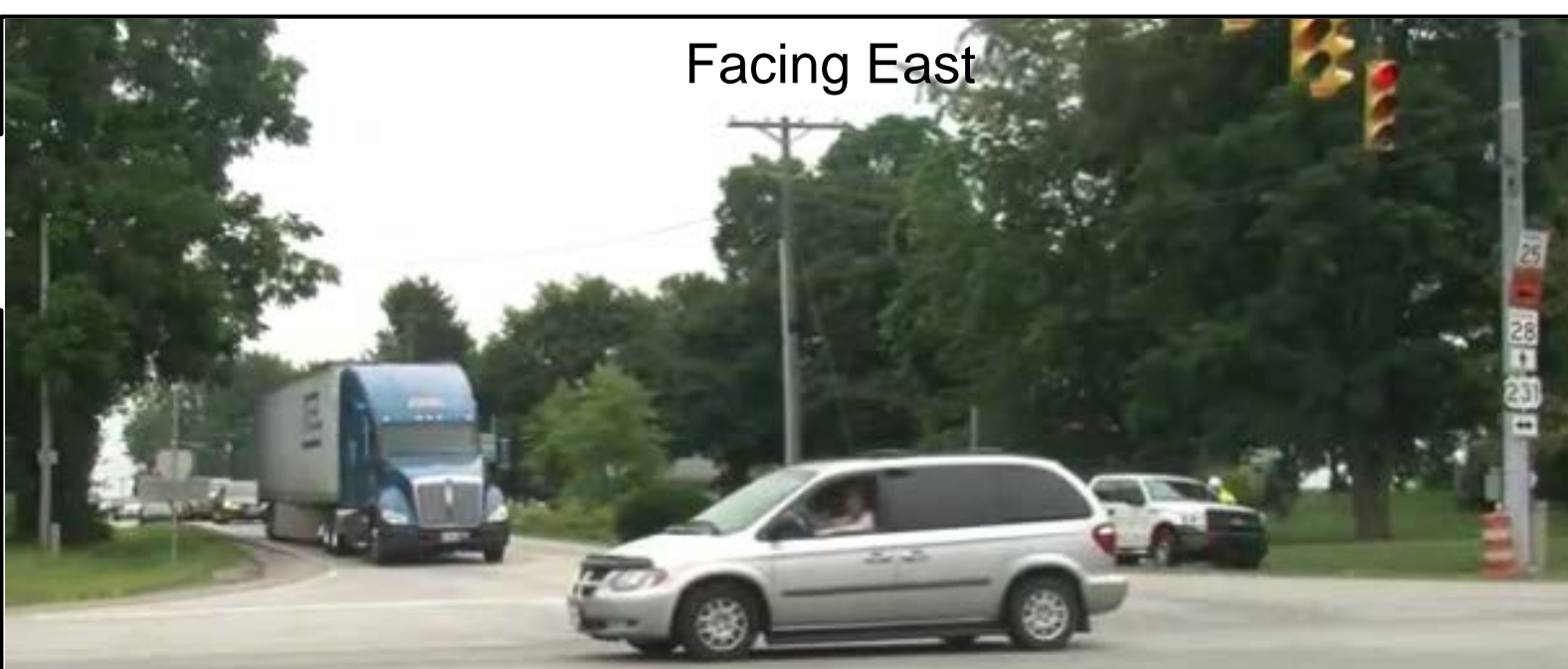




\section{Temporary Signal at US 231 \& SR 28 "All roads lead to Romney"}

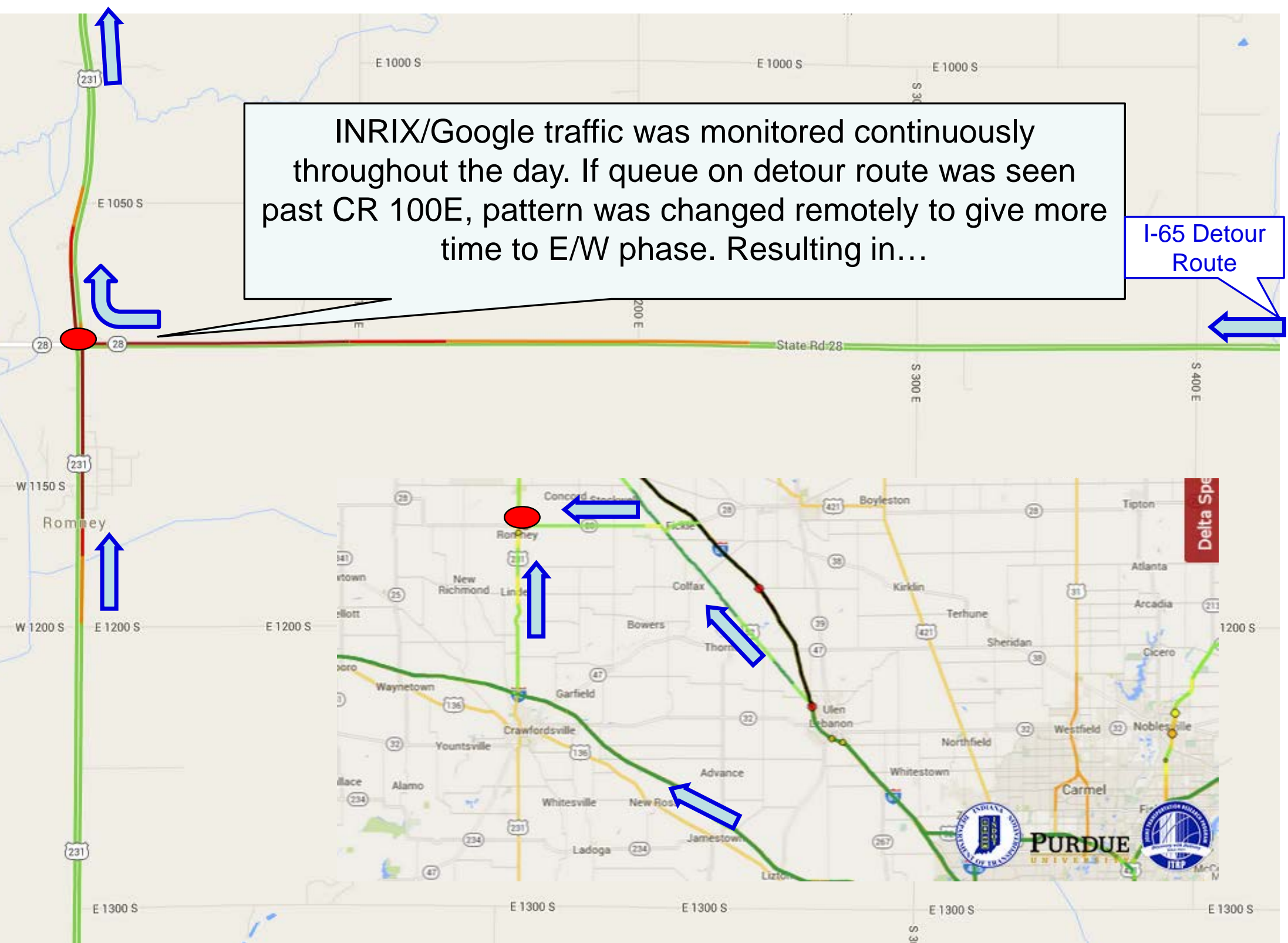




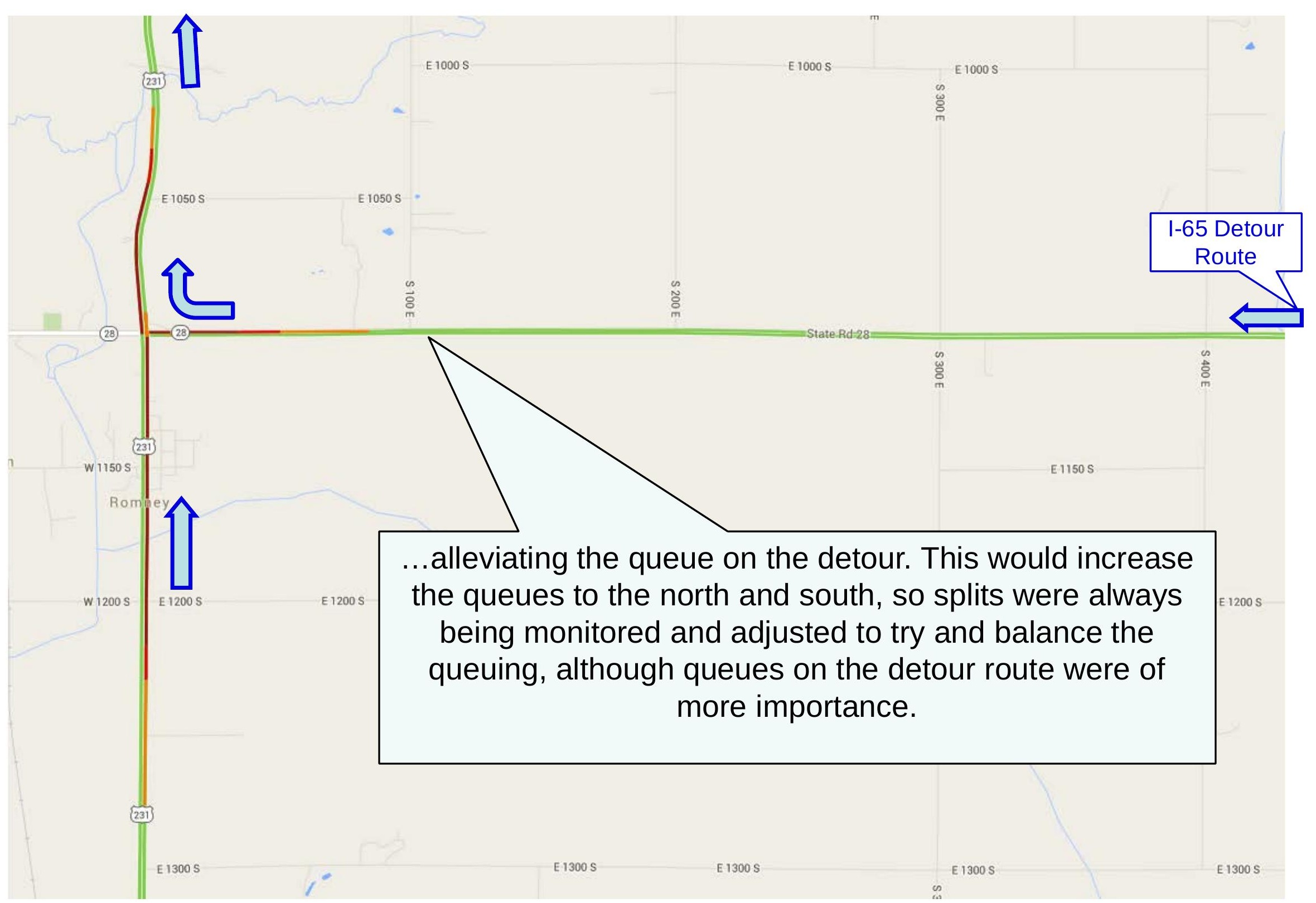




\section{Temporary Signal at US 52 \& SR 47}

- Safety concern at US 52 \& SR 47

- 2 way stop controlled E/W

- High crash history prior to closure

- Signal installed to increase safety

- Constructed overnight, $\sim 12$ hours

- Special detection installed to limit dilemma zone issues, red light running

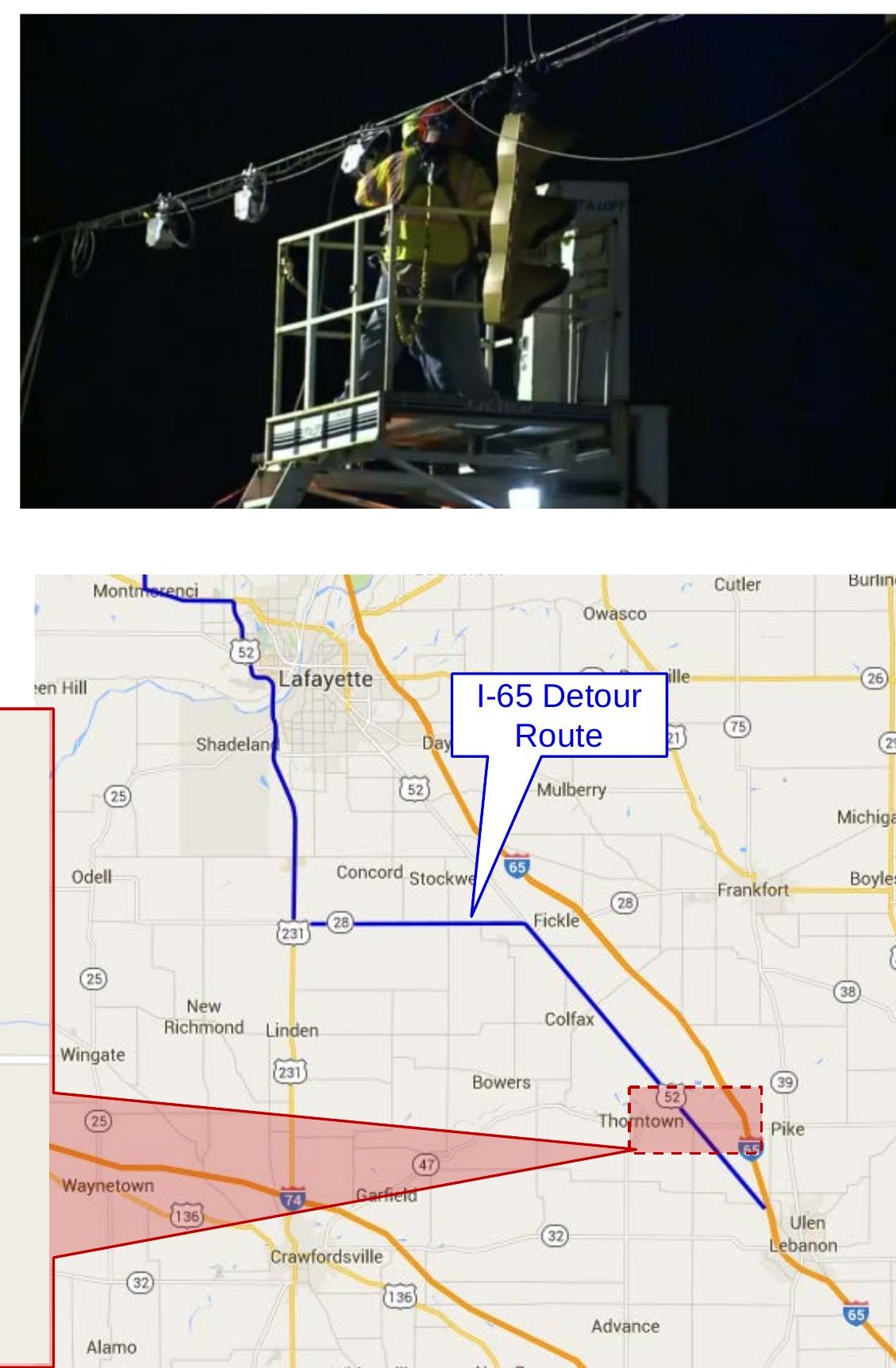




\section{Temporary Signal at US 52 \& SR 47}

- Installed speed sieve detection to extend phase safely for all vehicles travelling 40-70mph

- NB phase ran free with long min and longer max

- Eventually would gap out when no vehicles were approaching, serve SR 47 vehicles safely 
Before the Detour

\section{5 signal system coordinated only during AM and PM peaks.}

All 3 signals south of Wabash

River weren't running in coordination due to distance between signals and light volume on 231

\section{Was 2-way stop controlled E/W. A temporary 2-phase signal was constructed during the I-65 closure}


- All signals were retimed during the first week of the closure

- Coordinated every signal, only caring about northbound progression

- 150 second cycle length, heavily favoring northbound phase, (or westbound on north end)

- Adjusted offsets with goal of all signals having $>90 \%$ arrivals on green 

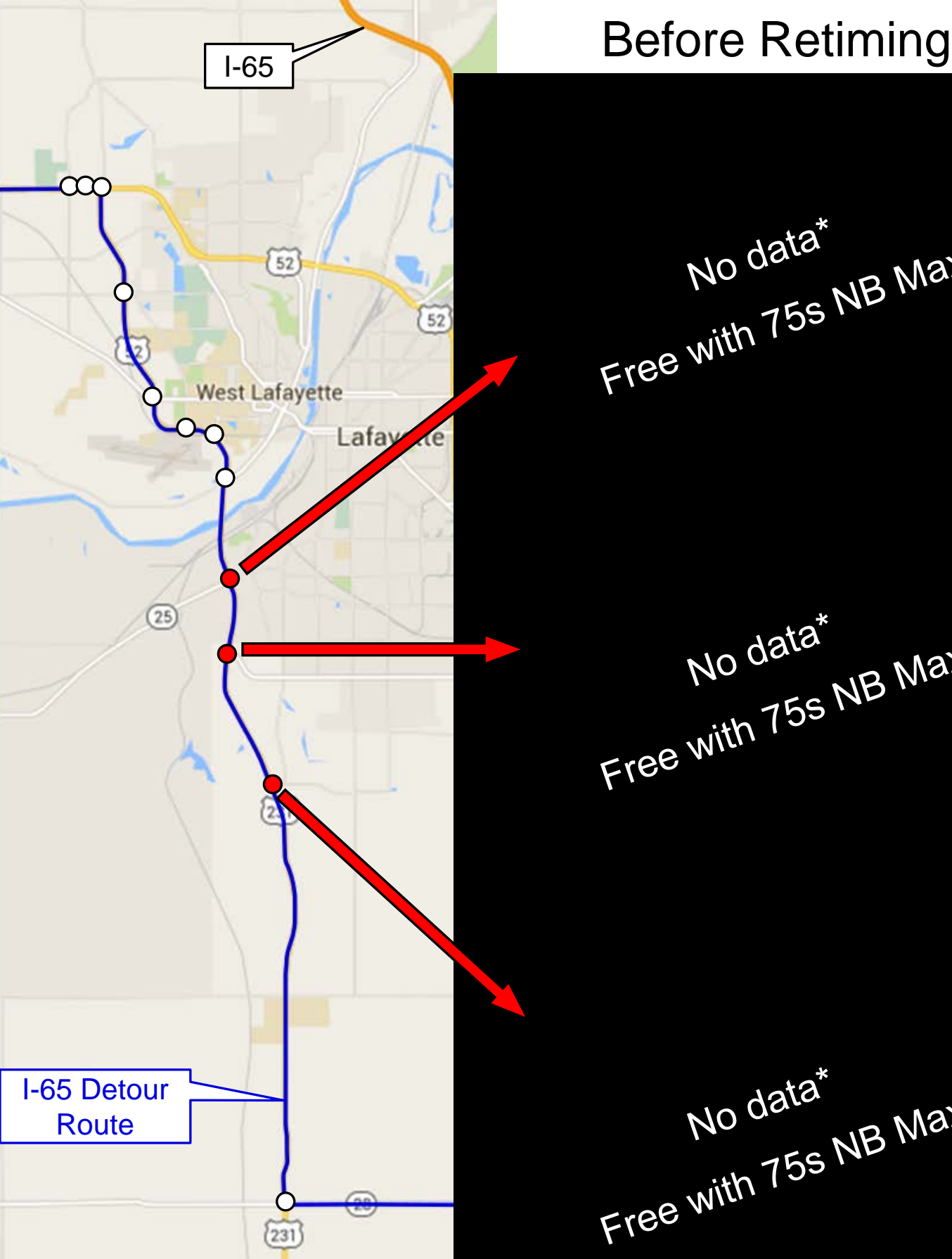

$\mathrm{O}=$ signal on detour

No data*
After Retiming

Free with 755 NB Max Free with 755 NB Max Free with 755 NB MaX

*Old controller prior to retiming
US 231 a SR 25

AOG: $8986 / 14130 \quad(63.6 \%)$
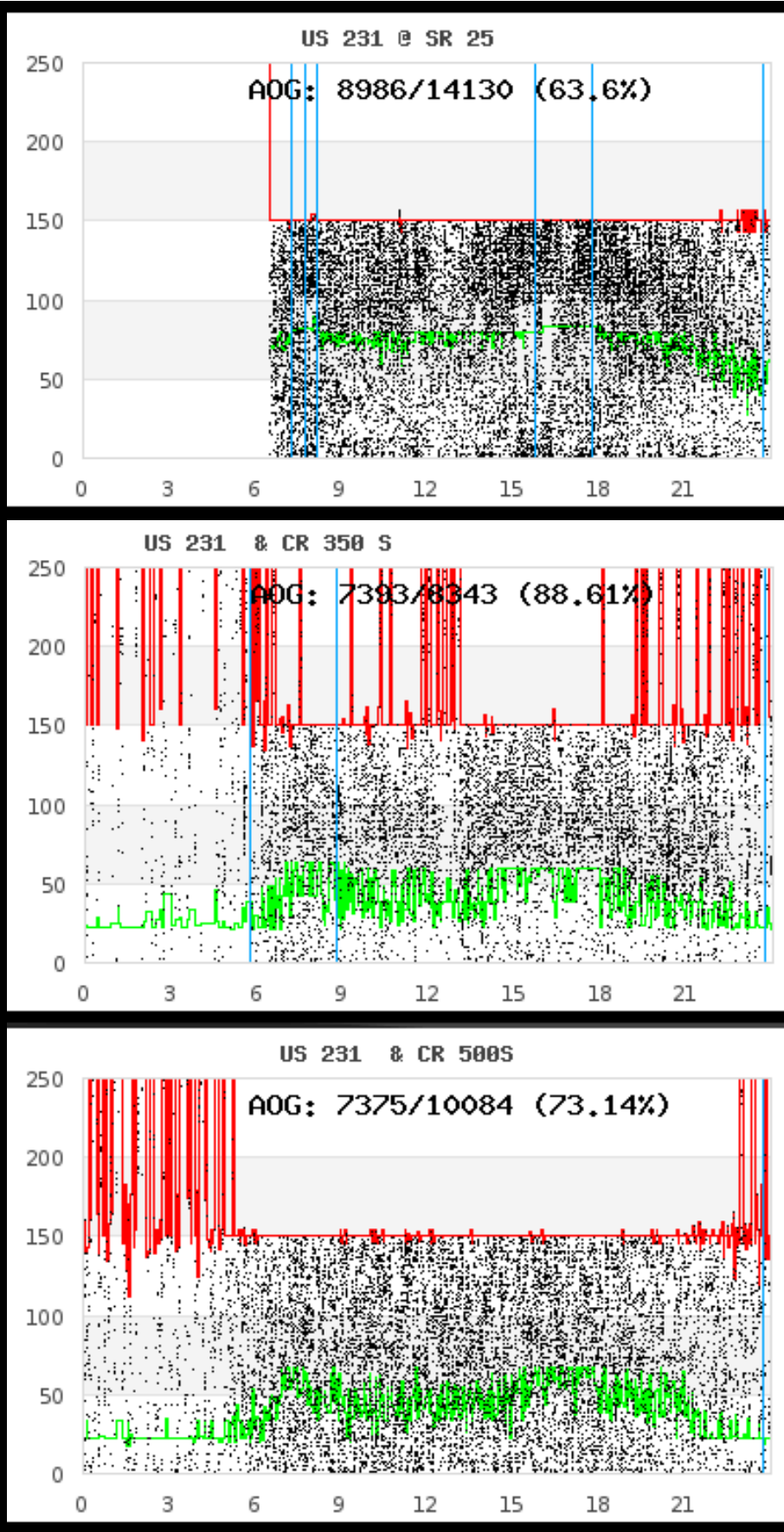


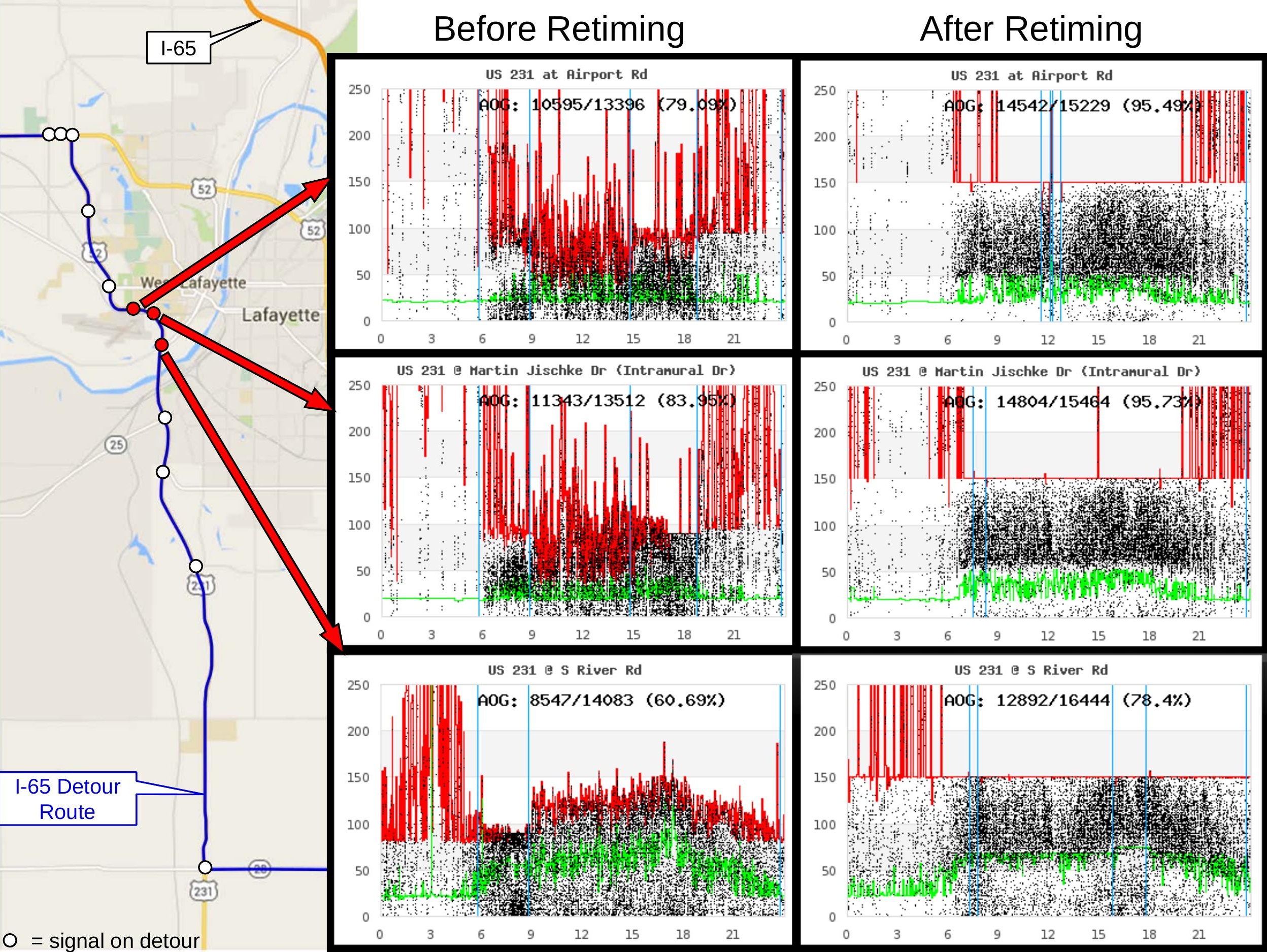



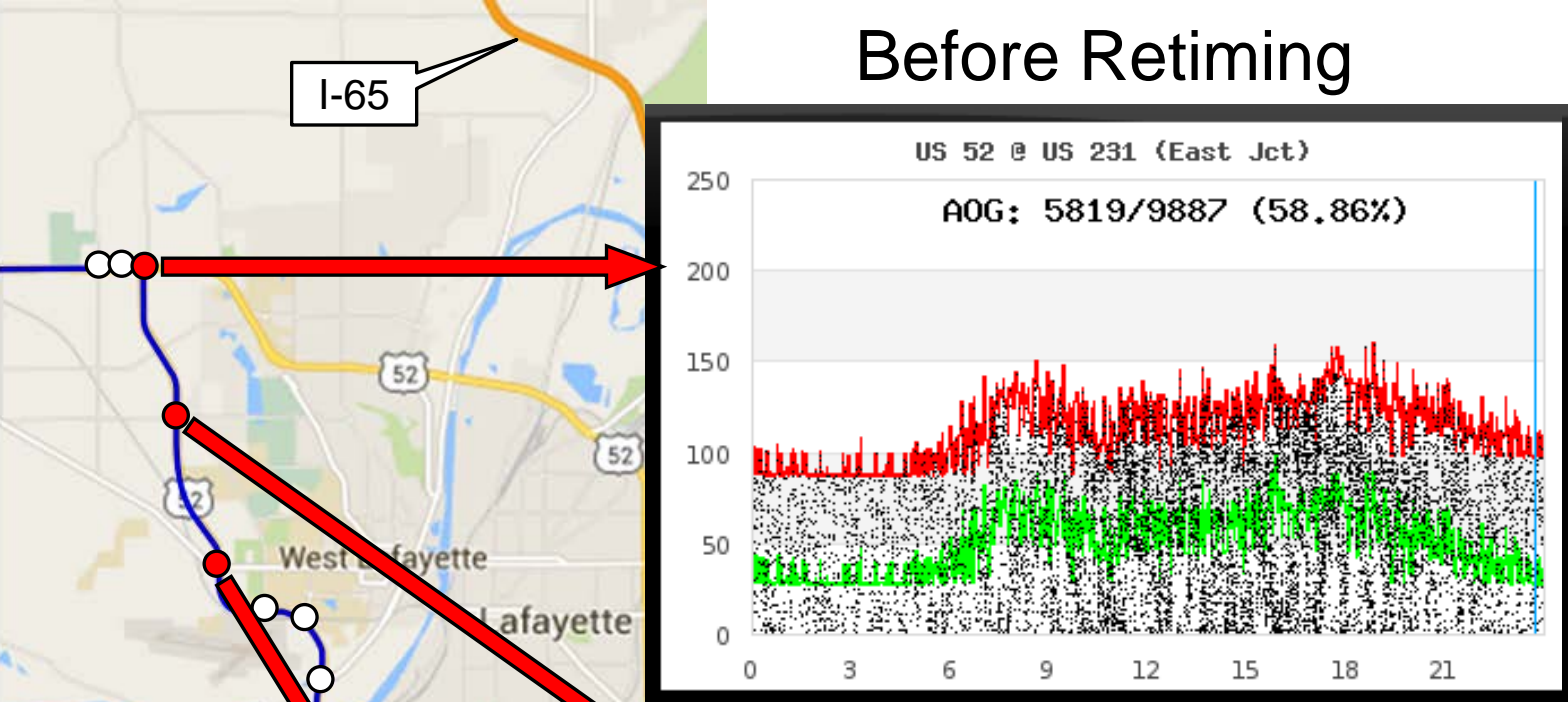

(25)

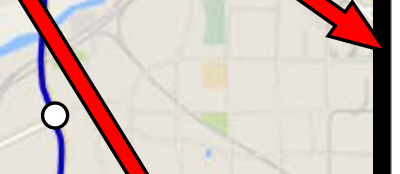

I-65 Detour Route

$\mathrm{O}=$ signal on detour

US 231 a Lindberg Rd (CR $200 \mathrm{~N}$ )

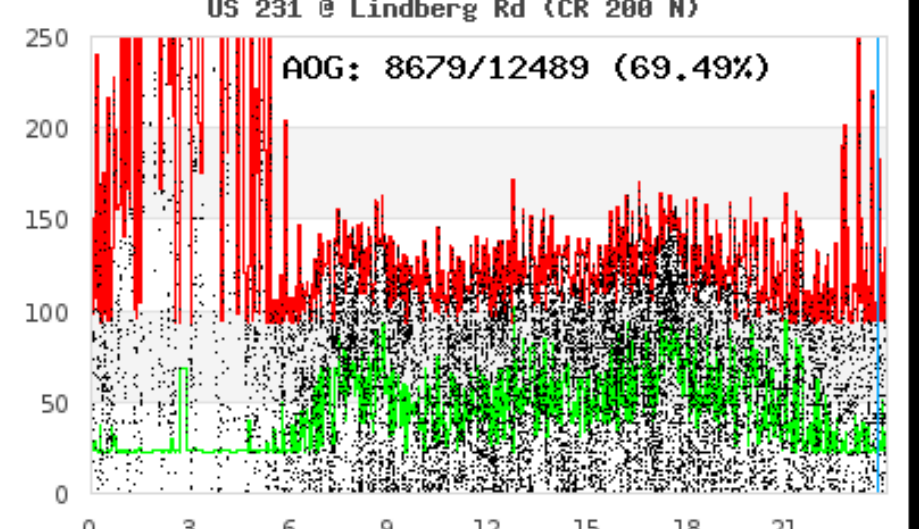

250

200

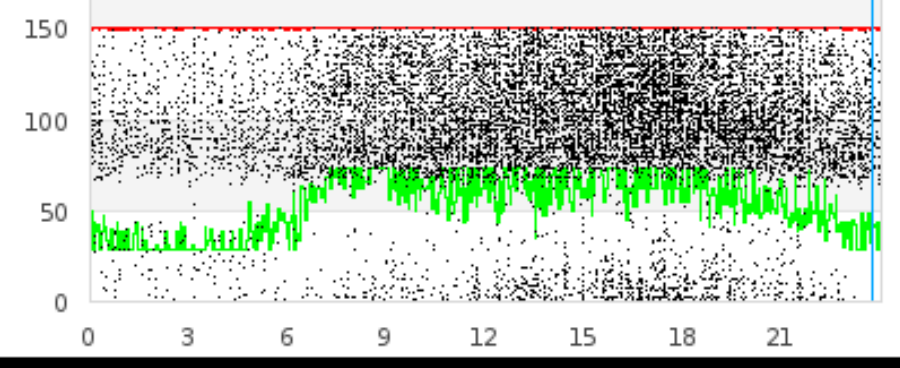

US 231 a Lindberg Rd (CR 200 N)

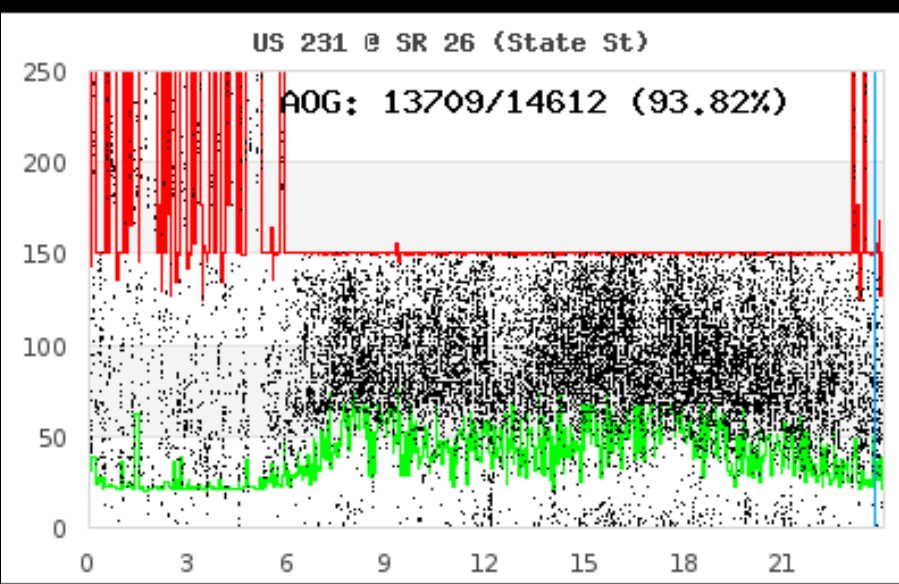

AOG: $9226 / 10242(90.08 \%)$

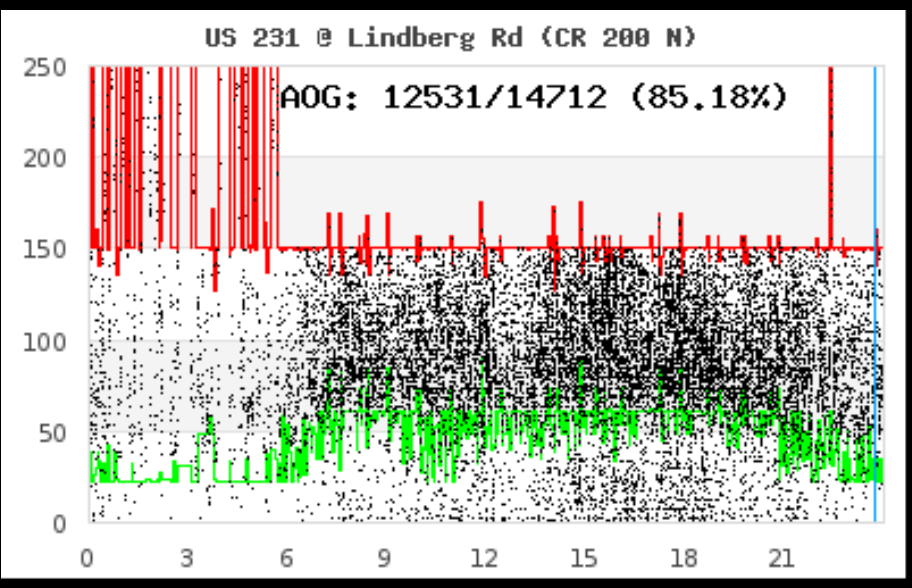

After Retiming

US 52 巳 US 231 (East Jct)

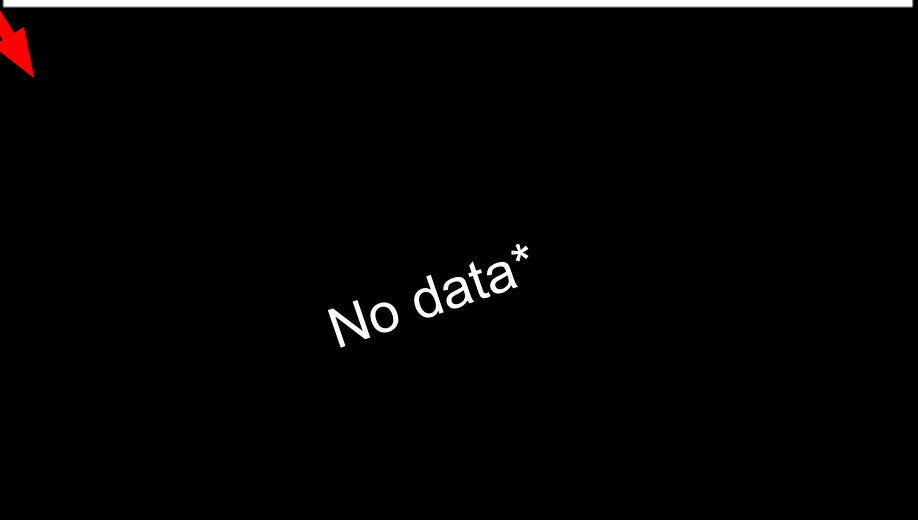

*Data logging issue corrected after retiming 


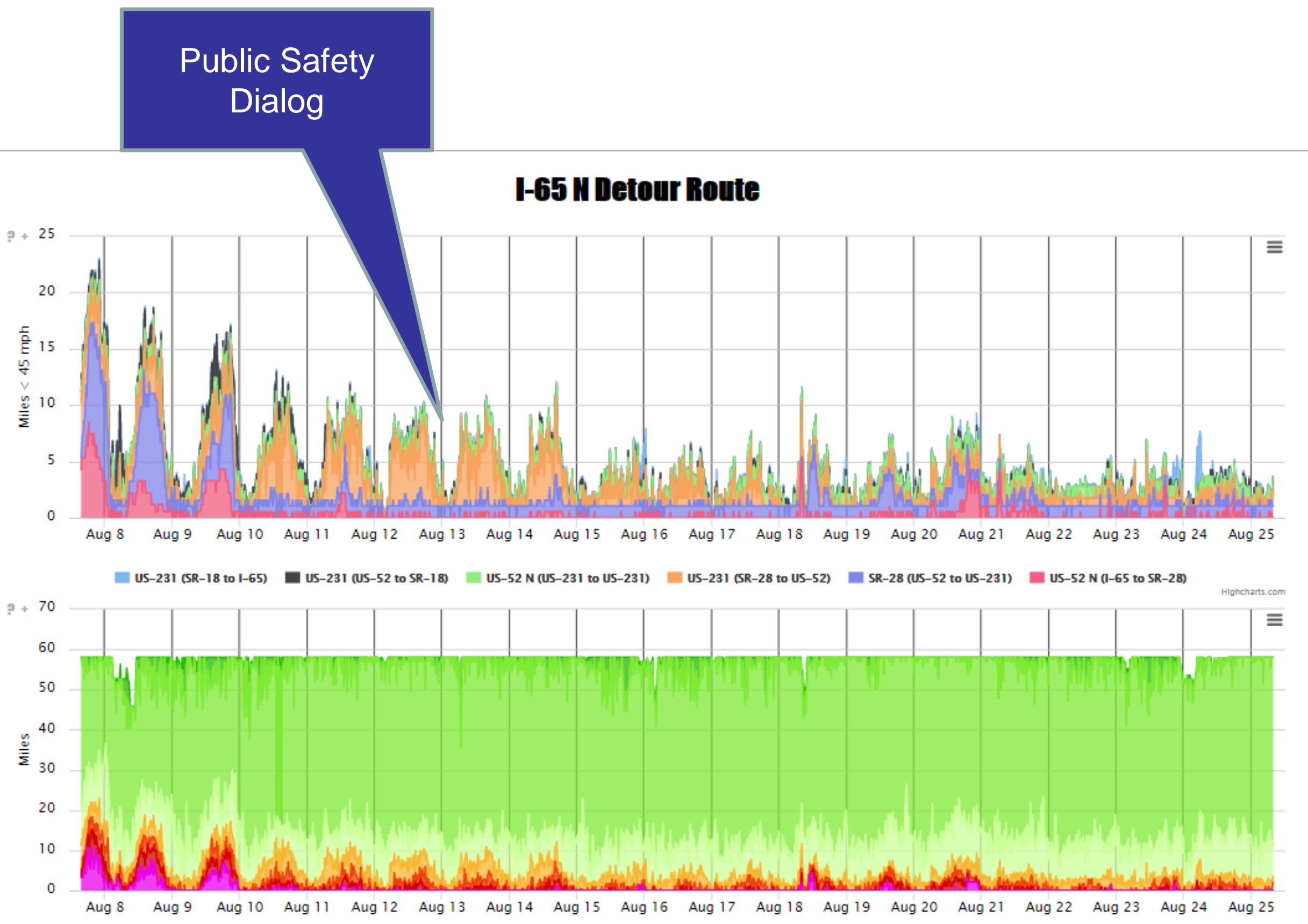




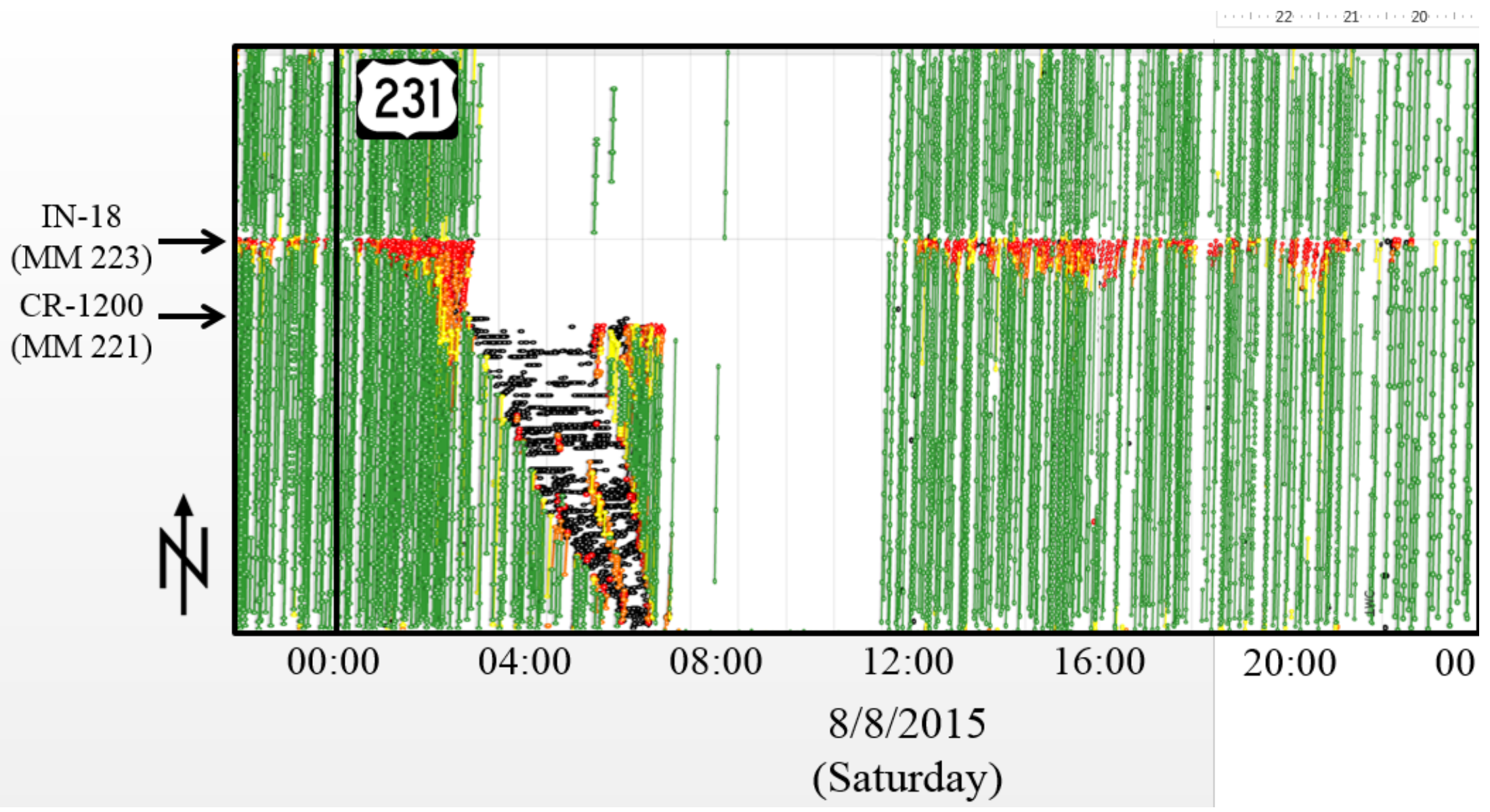




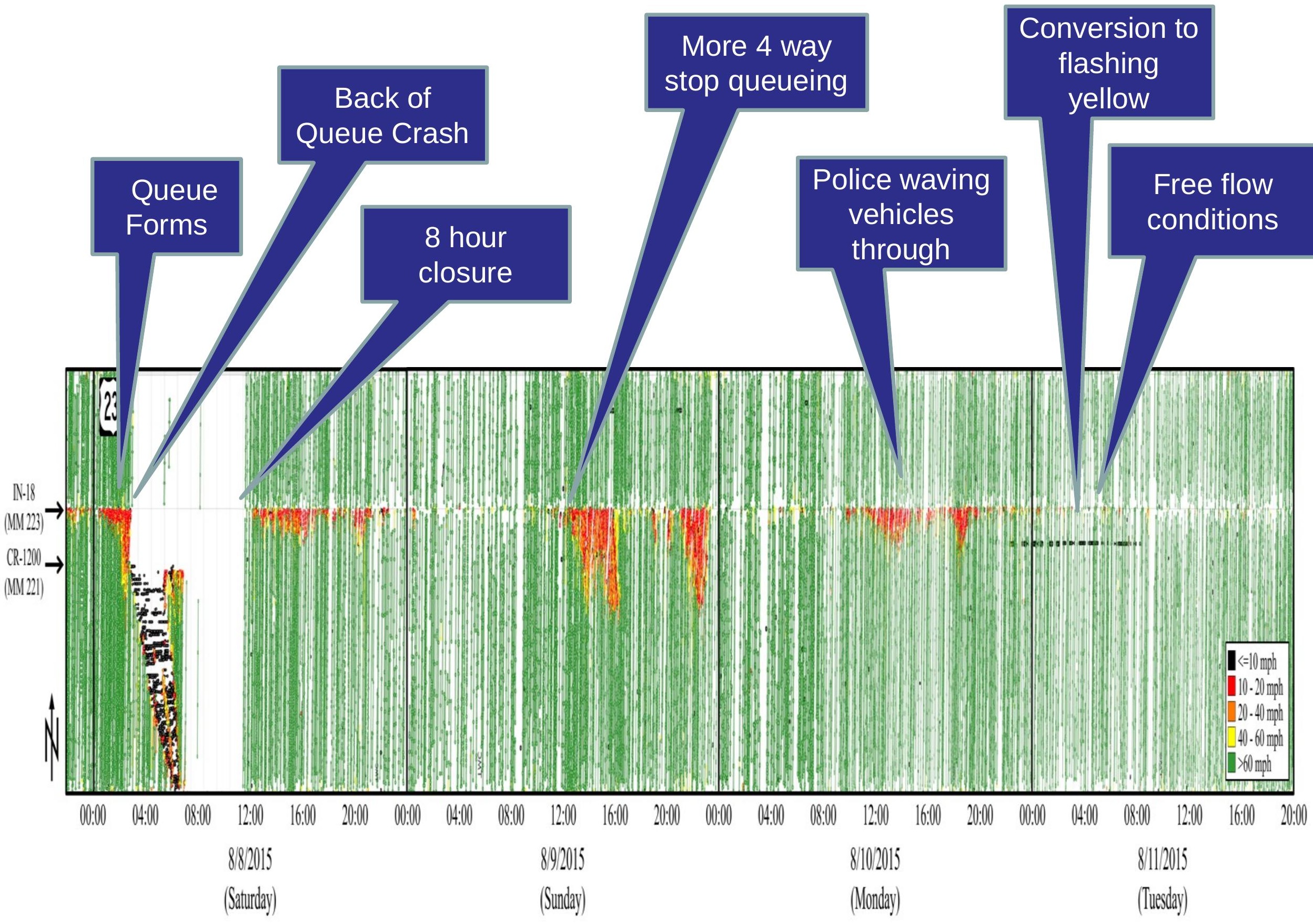




\section{Public Safety Workshop: August 13, 2015}
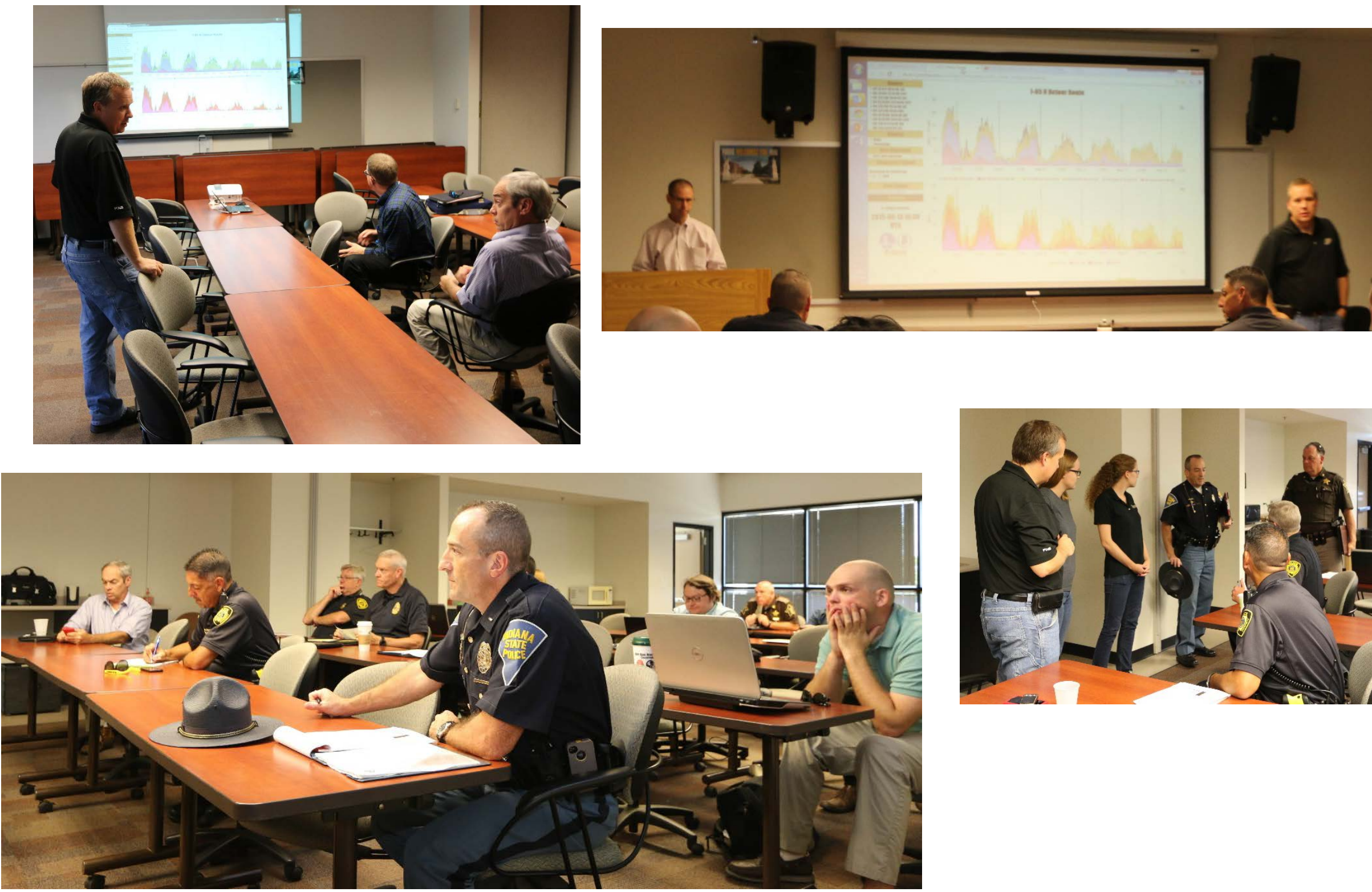


\section{I-65 Diversion Scenario}

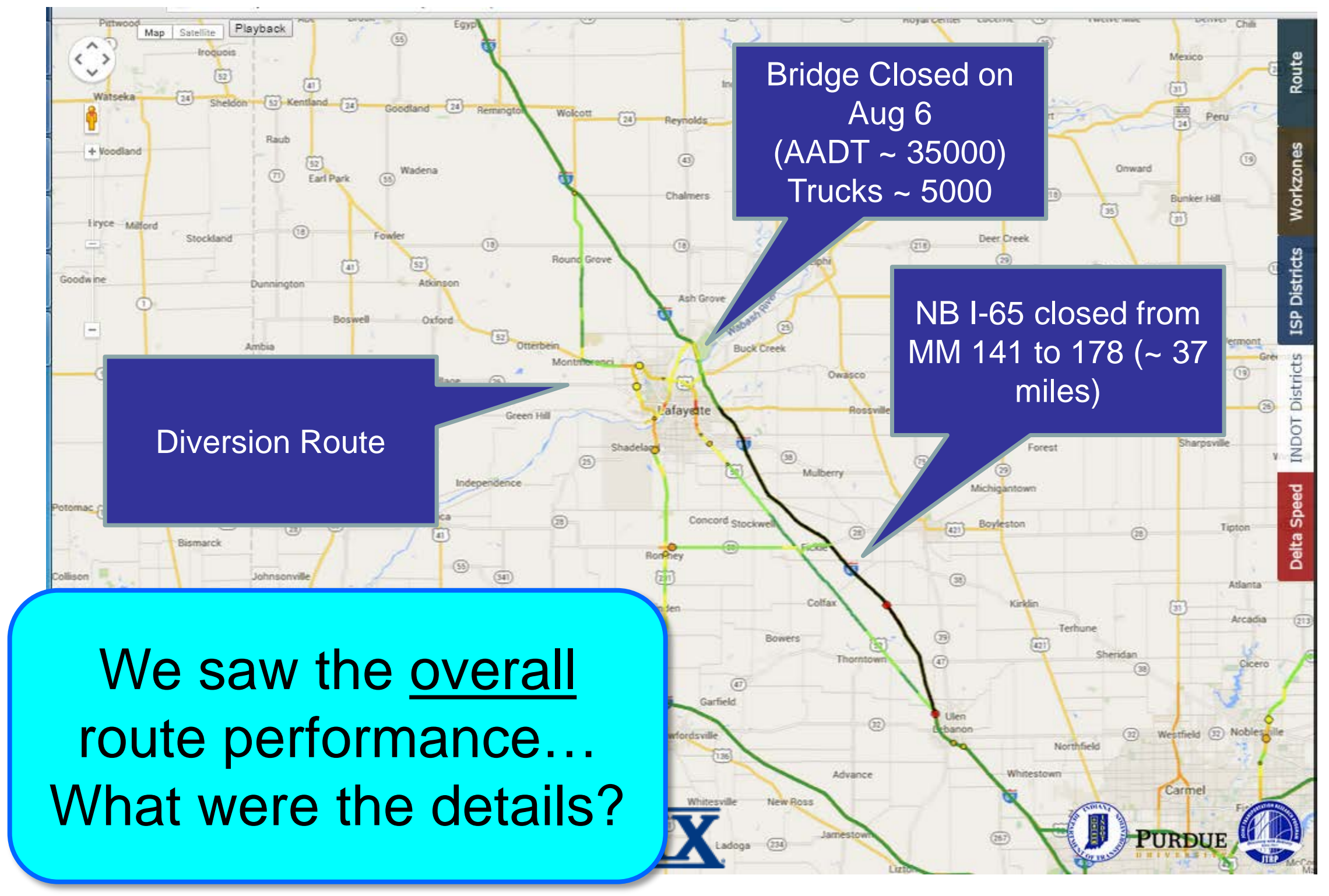




\section{Blue Tooth Data Collection Locations...}

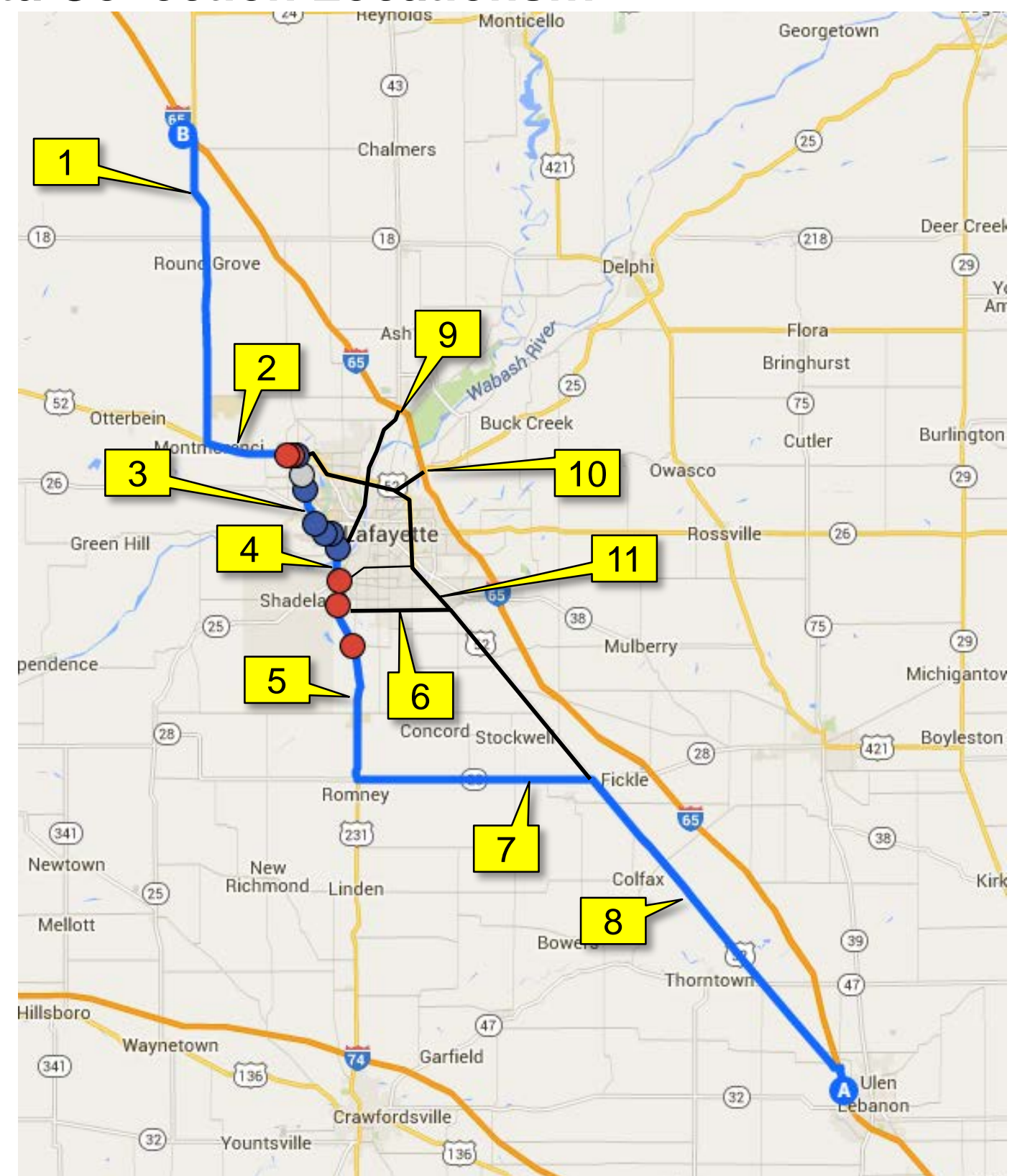




\section{Did Motorists Favor the Detour Route?}

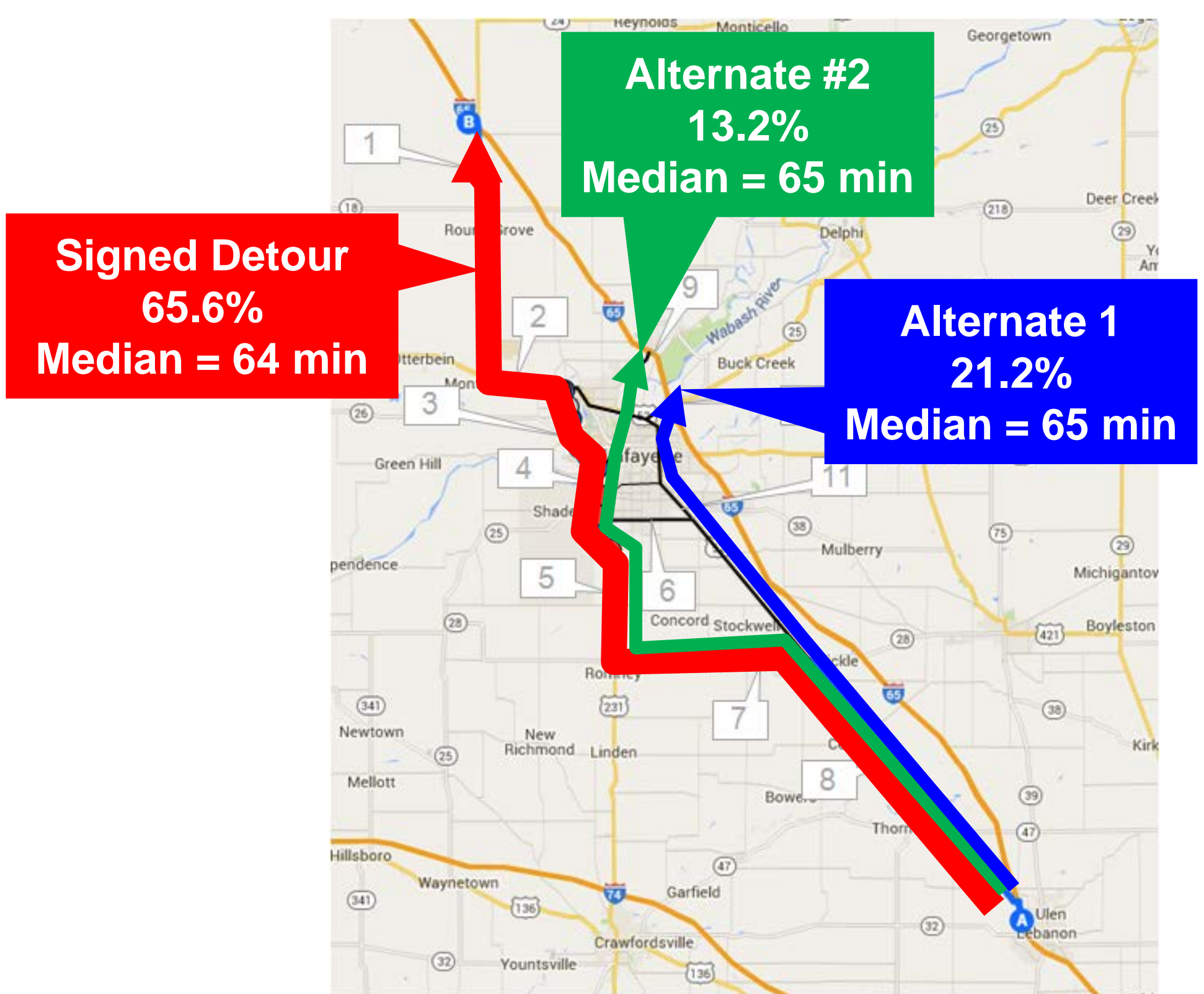




\section{Using Metrics to change the narrative.}

- Improvement to drive $\sim 60$

\section{Detour Diary: How bad 65 was the detour really?}

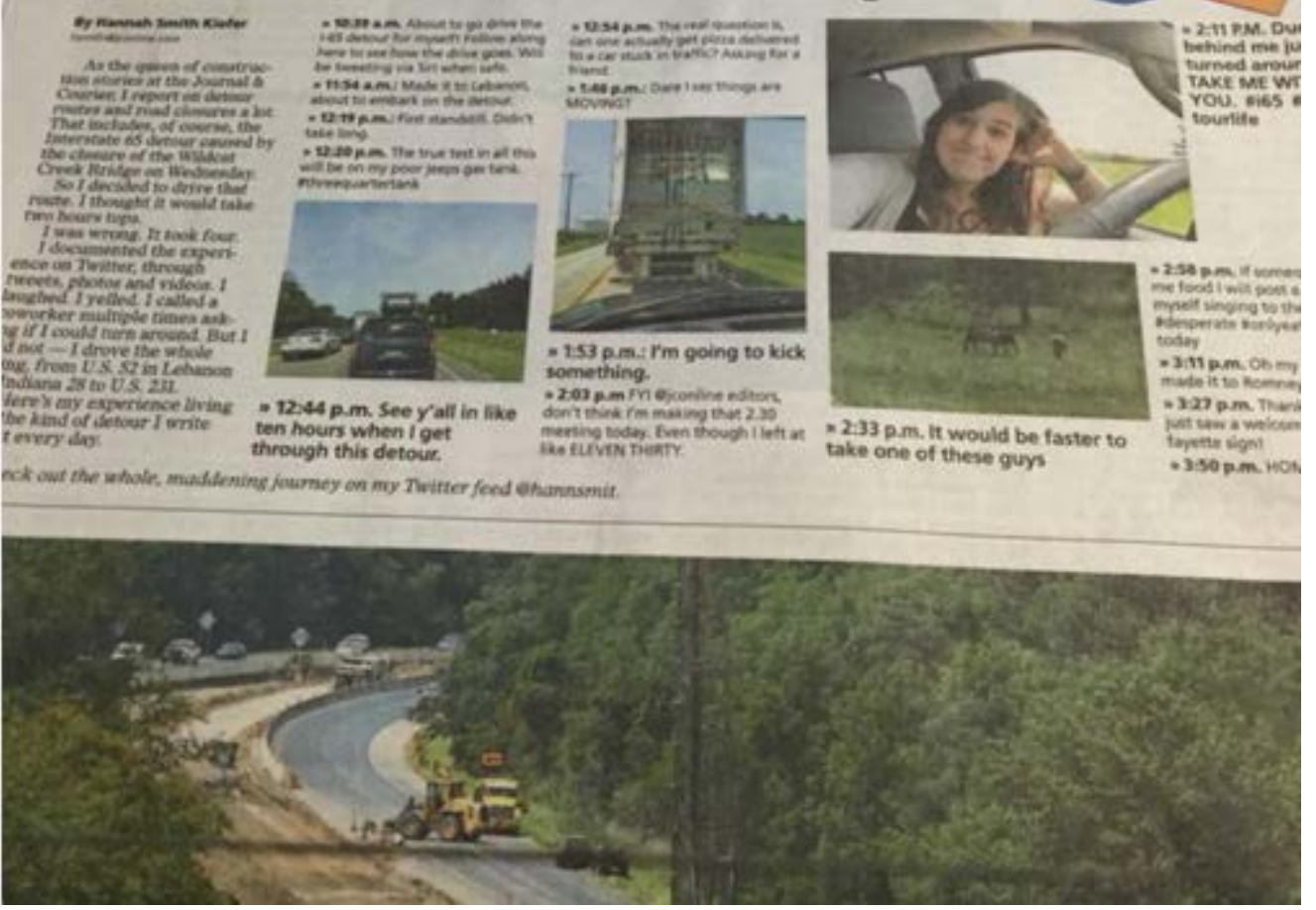
miles from 4 hours to about 64 minutes

- Bad news stories stopped being published

- Many reports of driving detour with zero to one stop!

- Media advocating use of the detour. 


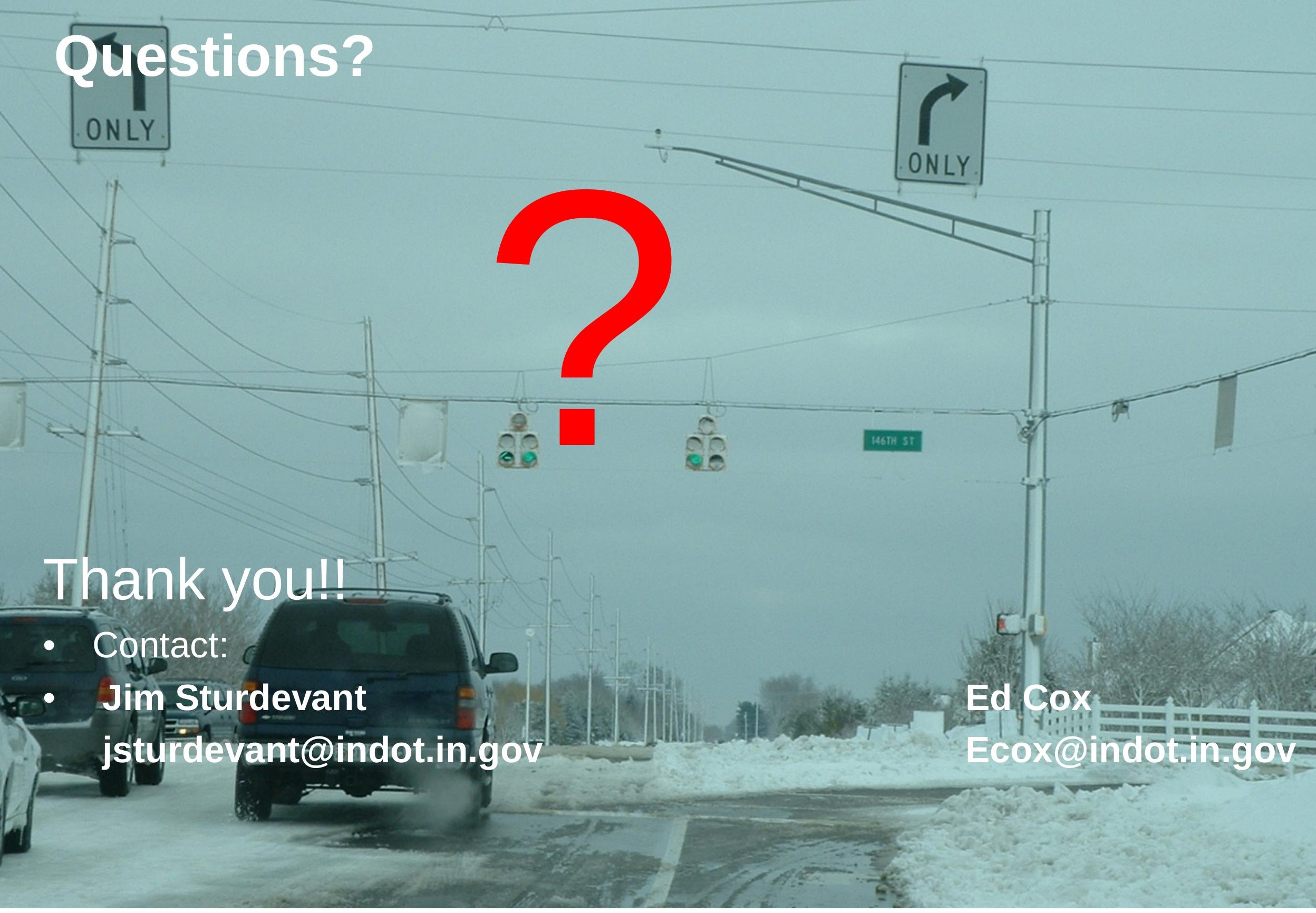

